\title{
A Straightforward Synthesis of 3-Acylphospholes
}

Magali Clochard, Joanna Grundy, Bruno Donnadieu, Francois Mathey

Supplementary Material: DFT calculations B3LYP/6-311+G(d,p)

\section{1-Formyl-2,5-dimethyl-1H-phosphole}

! Optimized Parameters !

! (Angstroms and Degrees) !

! Name Definition

Value Derivative Info.

! R1 R(1,2)

$1.3547 \quad-\mathrm{DE} / \mathrm{DX}=0.0$

! R2 $\mathrm{R}(1,7)$

1.828

$-\mathrm{DE} / \mathrm{DX}=0.0$

! R3 R(1,15)

1.4987

$-\mathrm{DE} / \mathrm{DX}=0.0$

! R4 R(2,3)

1.4572

$-\mathrm{DE} / \mathrm{DX}=0.0$

! R5 R(2,5)

1.0861

$-\mathrm{DE} / \mathrm{DX}=0.0$

! R6 R( $(3,4)$

1.3544

$-\mathrm{DE} / \mathrm{DX}=0.0$

! R7 R( $(3,6)$

1.0859

$-\mathrm{DE} / \mathrm{DX}=0.0$

! R8 R $(4,7)$

1.8239

$-\mathrm{DE} / \mathrm{DX}=0.0$

! R9 R(4,11)

1.4967

$-\mathrm{DE} / \mathrm{DX}=0.0$

! R10 R(7,8)

$1.8723-\mathrm{DE} / \mathrm{DX}=0.0$

! R11 R(8,9)

1.2039

$-\mathrm{DE} / \mathrm{DX}=0.0$

! R12 R(8,10)

$1.1119-\mathrm{DE} / \mathrm{DX}=0.0$

! R13 R(11,12)

$1.093-\mathrm{DE} / \mathrm{DX}=0.0$

! R14 R(11,13)

$1.0948-\mathrm{DE} / \mathrm{DX}=0.0$

! R15 R(11,14)

1.0948

$-\mathrm{DE} / \mathrm{DX}=0.0$

! R16 R(15,16)

$1.0964-\mathrm{DE} / \mathrm{DX}=0.0$

! R17 R(15,17)

1.093

$-\mathrm{DE} / \mathrm{DX}=0.0$

! R18 R(15,18)

1.0948

$-\mathrm{DE} / \mathrm{DX}=0.0$

! A1 A(2,1,7)

107.6936

$-\mathrm{DE} / \mathrm{DX}=0.0$

! A2 A $(2,1,15)$

127.1852

$-\mathrm{DE} / \mathrm{DX}=0.0$

! A3 A(7,1,15)

124.7848

$-\mathrm{DE} / \mathrm{DX}=0.0$ 


\begin{tabular}{|c|c|c|c|c|c|}
\hline ! A4 & $\mathrm{A}(1,2,3)$ & 115.7147 & $-\mathrm{DE} / \mathrm{DX}=0$ & 0.0 & $!$ \\
\hline ! A5 & $\mathrm{A}(1,2,5)$ & 122.6975 & $-\mathrm{DE} / \mathrm{DX}=0$ & 0.0 & $!$ \\
\hline ! A6 & $\mathrm{A}(3,2,5)$ & 121.5875 & $-\mathrm{DE} / \mathrm{DX}=0$ & 0.0 & $!$ \\
\hline ! A7 & $\mathrm{A}(2,3,4)$ & 115.7235 & $-\mathrm{DE} / \mathrm{DX}=0$ & 0.0 & $!$ \\
\hline ! A8 & $\mathrm{A}(2,3,6)$ & 121.6041 & $-\mathrm{DE} / \mathrm{DX}=0$ & 0.0 & $!$ \\
\hline ! A9 & $\mathrm{A}(4,3,6)$ & 122.6687 & $-\mathrm{DE} / \mathrm{DX}=0$ & 0.0 & $!$ \\
\hline ! A10 & $\mathrm{A}(3,4,7)$ & 107.8382 & $-\mathrm{DE} / \mathrm{DX}=$ & 0.0 & $!$ \\
\hline ! A11 & $\mathrm{A}(3,4,11)$ & 127.8298 & $-\mathrm{DE} / \mathrm{DX}=$ & 0.0 & $!$ \\
\hline ! A12 & $\mathrm{A}(7,4,11)$ & 124.2405 & $-\mathrm{DE} / \mathrm{DX}=$ & 0.0 & 1 \\
\hline ! A13 & $\mathrm{A}(1,7,4)$ & 92.2685 & $-\mathrm{DE} / \mathrm{DX}=0$ & 0.0 & $!$ \\
\hline ! A14 & $\mathrm{A}(1,7,8)$ & 102.7418 & $-\mathrm{DE} / \mathrm{DX}=$ & 0.0 & $!$ \\
\hline ! A15 & $\mathrm{A}(4,7,8)$ & 103.9732 & $-\mathrm{DE} / \mathrm{DX}=$ & 0.0 & $!$ \\
\hline ! A16 & $\mathrm{A}(7,8,9)$ & 124.7487 & $-\mathrm{DE} / \mathrm{DX}=$ & 0.0 & $!$ \\
\hline ! A17 & $\mathrm{A}(7,8,10)$ & 113.8403 & $-\mathrm{DE} / \mathrm{DX}=$ & 0.0 & $!$ \\
\hline ! A18 & $\mathrm{A}(9,8,10)$ & 121.2477 & $-\mathrm{DE} / \mathrm{DX}=$ & 0.0 & $!$ \\
\hline ! A19 & $\mathrm{A}(4,11,12)$ & 110.6231 & $-\mathrm{DE} / \mathrm{DX}=$ & 0.0 & $!$ \\
\hline ! A20 & $\mathrm{A}(4,11,13)$ & 110.7139 & $-\mathrm{DE} / \mathrm{DX}=$ & 0.0 & $!$ \\
\hline ! A21 & $\mathrm{A}(4,11,14)$ & 111.7124 & -DE/DX = & 0.0 & $!$ \\
\hline ! A22 & $\mathrm{A}(12,11,13)$ & 108.4547 & $-\mathrm{DE} / \mathrm{DX}=$ & $=0.0$ & 1 \\
\hline ! A23 & $\mathrm{A}(12,11,14)$ & 108.1354 & $-\mathrm{DE} / \mathrm{DX}=$ & $=0.0$ & \\
\hline ! A24 & $\mathrm{A}(13,11,14)$ & 107.0604 & $-\mathrm{DE} / \mathrm{DX}=$ & $=0.0$ & ! \\
\hline ! A25 & $\mathrm{A}(1,15,16)$ & 111.8747 & $-\mathrm{DE} / \mathrm{DX}=$ & 0.0 & $!$ \\
\hline ! A26 & $\mathrm{A}(1,15,17)$ & 110.5911 & $-\mathrm{DE} / \mathrm{DX}=$ & 0.0 & $!$ \\
\hline ! A27 & $\mathrm{A}(1,15,18)$ & 111.7266 & $-\mathrm{DE} / \mathrm{DX}=$ & 0.0 & $!$ \\
\hline ! A28 & $\mathrm{A}(16,15,17)$ & 107.586 & $-\mathrm{DE} / \mathrm{DX}=$ & 0.0 & $!$ \\
\hline ! A29 & $\mathrm{A}(16,15,18)$ & 106.8255 & $-\mathrm{DE} / \mathrm{DX}=$ & $=0.0$ & ! \\
\hline ! A30 & $\mathrm{A}(17,15,18)$ & 108.0279 & $-\mathrm{DE} / \mathrm{DX}=$ & $=0.0$ & ! \\
\hline ! D1 & $\mathrm{D}(7,1,2,3)$ & -6.1525 & $-\mathrm{DE} / \mathrm{DX}=0$ & 0.0 & $!$ \\
\hline ! D2 & $\mathrm{D}(7,1,2,5)$ & 173.6313 & $-\mathrm{DE} / \mathrm{DX}=$ & 0.0 & $!$ \\
\hline D3 & $\mathrm{D}(15,1,2,3)$ & -179.6971 & $-\mathrm{DE} / \mathrm{DX}=$ & 0.0 & $!$ \\
\hline 4 & $\mathrm{D}(15,1,2,5)$ & 0.0867 & $-\mathrm{DE} / \mathrm{DX}=0$ & 0.0 & $!$ \\
\hline
\end{tabular}




\begin{tabular}{|c|c|c|c|c|}
\hline ! D5 & $\mathrm{D}(2,1,7,4)$ & 7.7967 & $-\mathrm{DE} / \mathrm{DX}=0.0$ & $!$ \\
\hline ! D6 & $\mathrm{D}(2,1,7,8)$ & 112.672 & $-\mathrm{DE} / \mathrm{DX}=0.0$ & $!$ \\
\hline ! D7 & $\mathrm{D}(15,1,7,4)$ & -178.4644 & $-\mathrm{DE} / \mathrm{DX}=0.0$ & $!$ \\
\hline ! D8 & $\mathrm{D}(15,1,7,8)$ & -73.5891 & $-\mathrm{DE} / \mathrm{DX}=0.0$ & $!$ \\
\hline ! D9 & $\mathrm{D}(2,1,15,16)$ & -124.6004 & $-\mathrm{DE} / \mathrm{DX}=0.0$ & 1 \\
\hline ! D10 & $\mathrm{D}(2,1,15,17)$ & -4.6907 & $-\mathrm{DE} / \mathrm{DX}=0.0$ & $!$ \\
\hline ! D11 & $\mathrm{D}(2,1,15,18)$ & 115.68 & $-\mathrm{DE} / \mathrm{DX}=0.0$ & $!$ \\
\hline ! D12 & $\mathrm{D}(7,1,15,16)$ & 62.8932 & $-\mathrm{DE} / \mathrm{DX}=0.0$ & ! \\
\hline ! D13 & $\mathrm{D}(7,1,15,17)$ & -177.1971 & $-\mathrm{DE} / \mathrm{DX}=0.0$ & \\
\hline ! D14 & $\mathrm{D}(7,1,15,18)$ & -56.8264 & $-\mathrm{DE} / \mathrm{DX}=0.0$ & ! \\
\hline ! D15 & $\mathrm{D}(1,2,3,4)$ & 0.3298 & $-\mathrm{DE} / \mathrm{DX}=0.0$ & $!$ \\
\hline ! D16 & $\mathrm{D}(1,2,3,6)$ & -178.9895 & $-\mathrm{DE} / \mathrm{DX}=0.0$ & $!$ \\
\hline ! D17 & $\mathrm{D}(5,2,3,4)$ & -179.4566 & $-\mathrm{DE} / \mathrm{DX}=0.0$ & $!$ \\
\hline ! D18 & $\mathrm{D}(5,2,3,6)$ & 1.224 & $-\mathrm{DE} / \mathrm{DX}=0.0$ & $!$ \\
\hline ! D19 & $\mathrm{D}(2,3,4,7)$ & 5.699 & $-\mathrm{DE} / \mathrm{DX}=0.0$ & $!$ \\
\hline ! D20 & $\mathrm{D}(2,3,4,11)$ & -177.6959 & $-\mathrm{DE} / \mathrm{DX}=0.0$ & ! \\
\hline ! D21 & $\mathrm{D}(6,3,4,7)$ & -174.9896 & $-\mathrm{DE} / \mathrm{DX}=0.0$ & $!$ \\
\hline ! D22 & $\mathrm{D}(6,3,4,11)$ & 1.6155 & $-\mathrm{DE} / \mathrm{DX}=0.0$ & $!$ \\
\hline ! D23 & $\mathrm{D}(3,4,7,1)$ & -7.6308 & $-\mathrm{DE} / \mathrm{DX}=0.0$ & $!$ \\
\hline ! D24 & $\mathrm{D}(3,4,7,8)$ & -111.3591 & $-\mathrm{DE} / \mathrm{DX}=0.0$ & $!$ \\
\hline ! D25 & $\mathrm{D}(11,4,7,1)$ & 175.6127 & $-\mathrm{DE} / \mathrm{DX}=0.0$ & $!$ \\
\hline ! D26 & $\mathrm{D}(11,4,7,8)$ & 71.8843 & $-\mathrm{DE} / \mathrm{DX}=0.0$ & $!$ \\
\hline ! D27 & $\mathrm{D}(3,4,11,12)$ & 2.7376 & $-\mathrm{DE} / \mathrm{DX}=0.0$ & $!$ \\
\hline ! D28 & $\mathrm{D}(3,4,11,13)$ & 122.9969 & $-\mathrm{DE} / \mathrm{DX}=0.0$ & ! \\
\hline ! D29 & $\mathrm{D}(3,4,11,14)$ & -117.7818 & $-\mathrm{DE} / \mathrm{DX}=0.0$ & \\
\hline ! D30 & $\mathrm{D}(7,4,11,12)$ & 178.8276 & $-\mathrm{DE} / \mathrm{DX}=0.0$ & 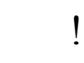 \\
\hline ! D31 & $\mathrm{D}(7,4,11,13)$ & -60.9131 & $-\mathrm{DE} / \mathrm{DX}=0.0$ & ! \\
\hline ! D32 & $\mathrm{D}(7,4,11,14)$ & 58.3081 & $-\mathrm{DE} / \mathrm{DX}=0.0$ & ! \\
\hline ! D33 & $\mathrm{D}(1,7,8,9)$ & -138.2332 & $-\mathrm{DE} / \mathrm{DX}=0.0$ & $!$ \\
\hline ! D34 & $\mathrm{D}(1,7,8,10)$ & 46.3794 & $-\mathrm{DE} / \mathrm{DX}=0.0$ & ! \\
\hline ! D35 & $\mathrm{D}(4,7,8,9)$ & -42.6042 & $-\mathrm{DE} / \mathrm{DX}=0.0$ & $!$ \\
\hline
\end{tabular}


! D36 $\mathrm{D}(4,7,8,10) \quad 142.0084 \quad-\mathrm{DE} / \mathrm{DX}=0.0 \quad$ !

GradGradGradGradGradGradGradGradGradGradGradGradGradGradGradGradGradGrad

Input orientation:

\begin{tabular}{|c|c|c|c|c|c|}
\hline \multirow{2}{*}{$\begin{array}{l}\text { Center } \\
\text { Number }\end{array}$} & \multicolumn{2}{|l|}{ Atomic } & Atomic & \multicolumn{2}{|c|}{ Coordinates (Angstron } \\
\hline & Numbe & & Type & $\mathrm{X}$ & $\mathrm{Z}$ \\
\hline 1 & 6 & 0 & -0.041357 & -0.036535 & 0.055279 \\
\hline 2 & 6 & 0 & 0.031915 & 0.290994 & 1.367736 \\
\hline 3 & 6 & 0 & 1.372655 & 0.322179 & 1.937711 \\
\hline 4 & 6 & 0 & 2.381517 & 0.026684 & 1.083683 \\
\hline 5 & 1 & 0 & -0.845670 & 0.520895 & 1.964977 \\
\hline 6 & 1 & 0 & 1.540690 & 0.557229 & 2.984517 \\
\hline 7 & 15 & & 1.666991 & -0.151052 & -0.584964 \\
\hline 8 & 6 & 0 & 1.845519 & -1.976499 & -0.961142 \\
\hline 9 & 8 & 0 & 2.824149 & -2.634523 & -0.718940 \\
\hline 10 & 1 & & 0.982977 & -2.407755 & -1.514628 \\
\hline 11 & 6 & & 3.843982 & -0.067350 & 1.387974 \\
\hline 12 & 1 & & 4.026606 & 0.093380 & 2.453580 \\
\hline 13 & 1 & & 4.233822 & -1.053084 & 1.114376 \\
\hline 14 & 1 & & 4.422776 & 0.673847 & 0.827445 \\
\hline 15 & 6 & & -1.278068 & -0.138782 & -0.785134 \\
\hline 16 & 1 & $v$ & -1.365726 & -1.120579 & -1.265154 \\
\hline 17 & 1 & & -2.171683 & 0.010904 & -0.173856 \\
\hline 18 & 1 & 0 & -1.286579 & 0.607475 & -1.586126 \\
\hline
\end{tabular}

Distance matrix (angstroms):

$\begin{array}{lllll}1 & 2 & 3 & 4 & 5\end{array}$ 


$$
\begin{aligned}
& 1 \mathrm{C} \quad 0.000000 \\
& \begin{array}{llll}
2 & \mathrm{C} & 1.354691 & 0.000000
\end{array} \\
& 3 \text { C } 2.381524 \quad 1.457198 \quad 0.000000 \\
& \begin{array}{llllll}
4 \mathrm{C} & 2.632856 & 2.381423 & 1.354432 & 0.000000
\end{array} \\
& \begin{array}{lllllll}
5 & \mathrm{H} & 2.145831 & 1.086143 & 2.227374 & 3.381665 & 0.000000
\end{array} \\
& \begin{array}{lllllll}
6 & \mathrm{H} & 3.381695 & 2.227389 & 1.085950 & 2.145143 & 2.595283
\end{array} \\
& \begin{array}{lllllll}
7 & \mathrm{P} & 1.827971 & 2.584940 & 2.583500 & 1.823875 & 3.642414
\end{array} \\
& \begin{array}{lllllll}
8 & \mathrm{C} & 2.890826 & 3.722144 & 3.729728 & 2.912275 & 4.694853
\end{array} \\
& \begin{array}{lllllll}
9 & \mathrm{O} & 3.944627 & 4.550762 & 4.231634 & 3.244595 & 5.534225
\end{array} \\
& \begin{array}{lllllll}
10 & \mathrm{H} & 3.022674 & 4.061501 & 4.418489 & 3.825393 & 4.901897
\end{array} \\
& \begin{array}{lllllll}
11 & \mathrm{C} & 4.107662 & 3.828926 & 2.561523 & 1.496743 & 4.761491
\end{array} \\
& \begin{array}{lllllll}
12 & \mathrm{H} & 4.724093 & 4.144354 & 2.713288 & 2.141818 & 4.915341
\end{array} \\
& \begin{array}{lllllll}
13 & \mathrm{H} & 4.520201 & 4.418909 & 3.279559 & 2.144266 & 5.385366
\end{array}
\end{aligned}
$$

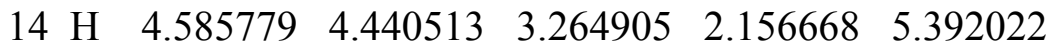

$$
\begin{aligned}
& \begin{array}{lllllll}
15 & \mathrm{C} & 1.498734 & 2.556485 & 3.827885 & 4.112471 & 2.860988
\end{array} \\
& \begin{array}{lllllll}
16 & \mathrm{H} & 2.161631 & 3.298189 & 4.454057 & 4.568925 & 3.660416
\end{array} \\
& \begin{array}{lllllll}
17 & \mathrm{H} & 2.143138 & 2.703849 & 4.137383 & 4.723694 & 2.567685
\end{array} \\
& \begin{array}{lllllll}
18 & \mathrm{H} & 2.158596 & 3.250214 & 4.423838 & 4.573853 & 3.579418
\end{array} \\
& \begin{array}{lllll}
6 & 7 & 8 & 9 & 10
\end{array} \\
& 7 \quad \mathrm{P} \quad 3.641265 \quad 0.000000 \\
& 8 \text { C } \quad 4.699034 \quad 1.872335 \quad 0.000000 \\
& \begin{array}{llllll}
9 & \mathrm{O} & 5.054715 & 2.743100 & 1.203900 & 0.000000
\end{array} \\
& \begin{array}{lllllll}
10 & \mathrm{H} & 5.417055 & 2.534731 & 1.111894 & 2.018529 & 0.000000
\end{array} \\
& \begin{array}{lllllll}
11 & \mathrm{C} & 2.871272 & 2.939181 & 3.627265 & 3.474123 & 4.699782
\end{array} \\
& \begin{array}{lllllll}
12 & \mathrm{H} & 2.583956 & 3.854904 & 4.549931 & 4.353418 & 5.591605
\end{array} \\
& \begin{array}{lllllll}
13 & \mathrm{H} & 3.652875 & 3.207810 & 3.296128 & 2.801638 & 4.394860
\end{array} \\
& 14 \mathrm{H} \quad 3.601802 \quad 3.204639 \quad 4.106779 \quad 3.986506 \quad 5.178205 \\
& \begin{array}{lllllll}
15 & \mathrm{C} & 4.758161 & 2.951879 & 3.628357 & 4.802217 & 3.285228
\end{array} \\
& 16 \mathrm{H} \quad 5.414979 \quad 3.255766 \quad 3.337231 \quad 4.488366 \quad 2.689881 \\
& \begin{array}{lllllll}
17 & \mathrm{H} & 4.904641 & 3.864020 & 4.550549 & 5.679237 & 4.195171
\end{array}
\end{aligned}
$$




\begin{tabular}{lcccccc}
18 & $\mathrm{H}$ & 5.374640 & 3.209558 & 4.108231 & 5.306661 & 3.774601 \\
& \multicolumn{7}{c}{11} & 12 & 13 & 14 & 15 & \\
11 & $\mathrm{C}$ & 0.000000 & & & & \\
12 & $\mathrm{H}$ & 1.093024 & 0.000000 & & & \\
13 & $\mathrm{H}$ & 1.094762 & 1.775046 & 0.000000 & & \\
14 & $\mathrm{H}$ & 1.094791 & 1.771499 & 1.760773 & 0.000000 & \\
15 & $\mathrm{C}$ & 5.564431 & 6.219545 & 5.901272 & 5.980000 & 0.000000 \\
16 & $\mathrm{H}$ & 5.940491 & 6.661826 & 6.084542 & 6.411372 & 1.096370 \\
17 & $\mathrm{H}$ & 6.215598 & 6.732682 & 6.619826 & 6.702909 & 1.092984 \\
18 & $\mathrm{H}$ & 5.968527 & 6.694286 & 6.365925 & 6.198909 & 1.094788
\end{tabular}

$\begin{array}{lll}16 & 17 & 18\end{array}$

$16 \mathrm{H} \quad 0.000000$

$17 \mathrm{H} \quad 1.766565 \quad 0.000000$

$\begin{array}{lllll}18 & \mathrm{H} & 1.759391 & 1.770257 & 0.000000\end{array}$

Stoichiometry C7H9OP

Zero-point correction $=\quad 0.140067($ Hartree/Particle $)$

Thermal correction to Energy $=\quad 0.150274$

Thermal correction to Enthalpy $=\quad 0.151218$

Thermal correction to Gibbs Free Energy $=\quad 0.104463$

Sum of electronic and zero-point Energies $=\quad-688.681212$

Sum of electronic and thermal Energies $=\quad-688.671005$

Sum of electronic and thermal Enthalpies $=\quad-688.670061$

Sum of electronic and thermal Free Energies $=\quad-688.716816$

\section{2-Formyl-2,5-dimethyl-2H-phosphole}

! Optimized Parameters !

! (Angstroms and Degrees) !

\begin{tabular}{|c|c|c|}
\hline ! Name Definition & Value & Derivative Info. \\
\hline ! R1 $\mathrm{R}(1,4)$ & 1.4518 & $-\mathrm{DE} / \mathrm{DX}=0.0$ \\
\hline
\end{tabular}




\begin{tabular}{|c|c|c|c|c|c|}
\hline ! R2 & $\mathrm{R}(1,7)$ & 1.7123 & $-\mathrm{DE} / \mathrm{DX}=$ & 0.0 & $!$ \\
\hline ! R3 & $\mathrm{R}(1,15)$ & 1.5012 & $-\mathrm{DE} / \mathrm{DX}=$ & 0.0 & $!$ \\
\hline ! R4 & $\mathrm{R}(2,3)$ & 1.4958 & $-\mathrm{DE} / \mathrm{DX}=$ & 0.0 & $!$ \\
\hline ! R5 & $\mathrm{R}(2,7)$ & 1.9075 & $-\mathrm{DE} / \mathrm{DX}=$ & 0.0 & $!$ \\
\hline ! R6 & $\mathrm{R}(2,8)$ & 1.5406 & $-\mathrm{DE} / \mathrm{DX}=$ & 0.0 & $!$ \\
\hline ! R7 & $\mathrm{R}(2,11)$ & 1.531 & $-\mathrm{DE} / \mathrm{DX}=$ & 0.0 & $!$ \\
\hline ! R8 & $\mathrm{R}(3,4)$ & 1.3499 & $-\mathrm{DE} / \mathrm{DX}=$ & 0.0 & $!$ \\
\hline ! R9 & $\mathrm{R}(3,5)$ & 1.0852 & $-\mathrm{DE} / \mathrm{DX}=$ & 0.0 & $!$ \\
\hline ! R10 & $\mathrm{R}(4,6)$ & 1.0861 & $-\mathrm{DE} / \mathrm{DX}=$ & 0.0 & $!$ \\
\hline ! R11 & $\mathrm{R}(8,9)$ & 1.2055 & $-\mathrm{DE} / \mathrm{DX}=$ & 0.0 & $!$ \\
\hline ! R12 & $\mathrm{R}(8,10)$ & 1.1077 & $-\mathrm{DE} / \mathrm{DX}=$ & 0.0 & $!$ \\
\hline ! R13 & $\mathrm{R}(11,12)$ & 1.0908 & $-\mathrm{DE} / \mathrm{DX}=$ & 0.0 & ! \\
\hline ! R14 & $\mathrm{R}(11,13)$ & 1.0936 & $-\mathrm{DE} / \mathrm{DX}=$ & 0.0 & ! \\
\hline ! R15 & $\mathrm{R}(11,14)$ & 1.0918 & $-\mathrm{DE} / \mathrm{DX}=$ & 0.0 & $!$ \\
\hline ! R16 & $\mathrm{R}(15,16)$ & 1.091 & $-\mathrm{DE} / \mathrm{DX}=$ & 0.0 & $!$ \\
\hline ! R17 & $\mathrm{R}(15,17)$ & 1.0958 & $-\mathrm{DE} / \mathrm{DX}=$ & 0.0 & $!$ \\
\hline ! R18 & $\mathrm{R}(15,18)$ & 1.0957 & $-\mathrm{DE} / \mathrm{DX}=$ & 0.0 & $!$ \\
\hline ! A1 & $\mathrm{A}(4,1,7)$ & 113.6858 & $-\mathrm{DE} / \mathrm{DX}=$ & 0.0 & ! \\
\hline ! A2 & $\mathrm{A}(4,1,15)$ & 121.0796 & $-\mathrm{DE} / \mathrm{DX}=$ & 0.0 & \\
\hline ! A3 & $\mathrm{A}(7,1,15)$ & 125.2203 & $-\mathrm{DE} / \mathrm{DX}=$ & 0.0 & \\
\hline ! A4 & $\mathrm{A}(3,2,7)$ & 105.6183 & $-\mathrm{DE} / \mathrm{DX}=$ & 0.0 & ! \\
\hline ! A5 & $\mathrm{A}(3,2,8)$ & 105.8694 & $-\mathrm{DE} / \mathrm{DX}=$ & 0.0 & ! \\
\hline ! A6 & $\mathrm{A}(3,2,11)$ & 115.6016 & $-\mathrm{DE} / \mathrm{DX}=$ & 0.0 & \\
\hline ! A7 & $\mathrm{A}(7,2,8)$ & 101.9636 & $-\mathrm{DE} / \mathrm{DX}=$ & 0.0 & ! \\
\hline ! A8 & $\mathrm{A}(7,2,11)$ & 114.8104 & $-\mathrm{DE} / \mathrm{DX}=$ & 0.0 & \\
\hline ! A9 & $\mathrm{A}(8,2,11)$ & 111.7141 & $-\mathrm{DE} / \mathrm{DX}=$ & 0.0 & \\
\hline ! A10 & $\mathrm{A}(2,3,4)$ & 114.8716 & $-\mathrm{DE} / \mathrm{DX}=$ & 0.0 & \\
\hline ! A11 & $\mathrm{A}(2,3,5)$ & 120.7949 & $-\mathrm{DE} / \mathrm{DX}=$ & 0.0 & \\
\hline ! A12 & $\mathrm{A}(4,3,5)$ & 124.2652 & $-\mathrm{DE} / \mathrm{DX}=$ & 0.0 & \\
\hline ! A13 & $\mathrm{A}(1,4,3)$ & 114.5814 & $-\mathrm{DE} / \mathrm{DX}=$ & 0.0 & \\
\hline A14 & $\mathrm{A}(1,4,6)$ & 121.7733 & $-\mathrm{DE} / \mathrm{DX}=$ & 0.0 & \\
\hline
\end{tabular}




\begin{tabular}{|c|c|c|c|c|}
\hline ! A15 & $\mathrm{A}(3,4,6)$ & 123.6404 & $-\mathrm{DE} / \mathrm{DX}=0.0$ & $!$ \\
\hline ! A16 & $\mathrm{A}(1,7,2)$ & 90.964 & $-\mathrm{DE} / \mathrm{DX}=0.0$ & $!$ \\
\hline ! A17 & $\mathrm{A}(2,8,9)$ & 124.3344 & $-\mathrm{DE} / \mathrm{DX}=0.0$ & $!$ \\
\hline ! A18 & $\mathrm{A}(2,8,10)$ & 114.056 & $-\mathrm{DE} / \mathrm{DX}=0.0$ & $!$ \\
\hline ! A19 & $\mathrm{A}(9,8,10)$ & 121.6074 & $-\mathrm{DE} / \mathrm{DX}=0.0$ & $!$ \\
\hline ! A20 & $\mathrm{A}(2,11,12)$ & 110.7702 & $-\mathrm{DE} / \mathrm{DX}=0.0$ & $!$ \\
\hline ! A21 & $\mathrm{A}(2,11,13)$ & 110.2959 & $-\mathrm{DE} / \mathrm{DX}=0.0$ & $!$ \\
\hline ! A22 & $\mathrm{A}(2,11,14)$ & 110.6988 & $-\mathrm{DE} / \mathrm{DX}=0.0$ & $!$ \\
\hline ! A23 & $\mathrm{A}(12,11,13)$ & 107.3861 & $-\mathrm{DE} / \mathrm{DX}=0.0$ & ! \\
\hline ! A24 & $\mathrm{A}(12,11,14)$ & 108.8846 & $-\mathrm{DE} / \mathrm{DX}=0.0$ & ! \\
\hline ! A25 & $\mathrm{A}(13,11,14)$ & 108.7159 & $-\mathrm{DE} / \mathrm{DX}=0.0$ & ! \\
\hline ! A26 & $\mathrm{A}(1,15,16)$ & 112.1655 & $-\mathrm{DE} / \mathrm{DX}=0.0$ & $!$ \\
\hline ! A27 & $\mathrm{A}(1,15,17)$ & 110.6061 & $-\mathrm{DE} / \mathrm{DX}=0.0$ & $!$ \\
\hline ! A28 & $\mathrm{A}(1,15,18)$ & 110.6071 & $-\mathrm{DE} / \mathrm{DX}=0.0$ & $!$ \\
\hline ! A29 & $\mathrm{A}(16,15,17)$ & 108.2853 & $-\mathrm{DE} / \mathrm{DX}=0.0$ & ! \\
\hline ! A30 & $\mathrm{A}(16,15,18)$ & 108.0452 & $-\mathrm{DE} / \mathrm{DX}=0.0$ & $!$ \\
\hline ! A31 & $\mathrm{A}(17,15,18)$ & 106.9434 & $-\mathrm{DE} / \mathrm{DX}=0.0$ & ! \\
\hline ! D1 & $\mathrm{D}(7,1,4,3)$ & 0.1636 & $-\mathrm{DE} / \mathrm{DX}=0.0$ & $!$ \\
\hline ! D2 & $\mathrm{D}(7,1,4,6)$ & -179.056 & $-\mathrm{DE} / \mathrm{DX}=0.0$ & $!$ \\
\hline ! D3 & $\mathrm{D}(15,1,4,3)$ & 178.8547 & $-\mathrm{DE} / \mathrm{DX}=0.0$ & $!$ \\
\hline ! D4 & $\mathrm{D}(15,1,4,6)$ & -0.3649 & $-\mathrm{DE} / \mathrm{DX}=0.0$ & $!$ \\
\hline ! D5 & $\mathrm{D}(4,1,7,2)$ & -2.8843 & $-\mathrm{DE} / \mathrm{DX}=0.0$ & $!$ \\
\hline ! D6 & $\mathrm{D}(15,1,7,2)$ & 178.488 & $-\mathrm{DE} / \mathrm{DX}=0.0$ & $!$ \\
\hline ! D7 & $\mathrm{D}(4,1,15,16)$ & -177.2636 & $-\mathrm{DE} / \mathrm{DX}=0.0$ & $!$ \\
\hline ! D8 & $\mathrm{D}(4,1,15,17)$ & -56.2582 & $-\mathrm{DE} / \mathrm{DX}=0.0$ & $!$ \\
\hline ! D9 & $\mathrm{D}(4,1,15,18)$ & 62.0368 & $-\mathrm{DE} / \mathrm{DX}=0.0$ & $!$ \\
\hline ! D10 & $\mathrm{D}(7,1,15,16)$ & 1.2691 & $-\mathrm{DE} / \mathrm{DX}=0.0$ & $!$ \\
\hline ! D11 & $\mathrm{D}(7,1,15,17)$ & 122.2745 & $-\mathrm{DE} / \mathrm{DX}=0.0$ & ! \\
\hline ! D12 & $\mathrm{D}(7,1,15,18)$ & -119.4305 & $-\mathrm{DE} / \mathrm{DX}=0.0$ & ! \\
\hline ! D13 & $\mathrm{D}(7,2,3,4)$ & -5.4751 & $-\mathrm{DE} / \mathrm{DX}=0.0$ & $!$ \\
\hline D14 & $\mathrm{D}(7,2,3,5)$ & 177.4045 & $-\mathrm{DE} / \mathrm{DX}=0.0$ & $!$ \\
\hline
\end{tabular}




\begin{tabular}{|c|c|c|c|c|c|}
\hline ! D15 & $\mathrm{D}(8,2,3,4)$ & 102.1769 & $-\mathrm{DE} / \mathrm{DX}=$ & 0.0 & \\
\hline ! D16 & $\mathrm{D}(8,2,3,5)$ & -74.9434 & $-\mathrm{DE} / \mathrm{DX}=$ & 0.0 & \\
\hline ! D17 & $\mathrm{D}(11,2,3,4)$ & -133.5787 & $-\mathrm{DE} / \mathrm{DX}=$ & 0.0 & \\
\hline ! D18 & $\mathrm{D}(11,2,3,5)$ & 49.301 & $-\mathrm{DE} / \mathrm{DX}=$ & 0.0 & \\
\hline ! D19 & $\mathrm{D}(3,2,7,1)$ & 4.5424 & $-\mathrm{DE} / \mathrm{DX}=0$ & 0.0 & \\
\hline ! D20 & $\mathrm{D}(8,2,7,1)$ & -105.9124 & $-\mathrm{DE} / \mathrm{DX}=$ & 0.0 & \\
\hline ! D21 & $\mathrm{D}(11,2,7,1)$ & 133.1169 & -DE/DX = & 0.0 & \\
\hline ! D22 & $\mathrm{D}(3,2,8,9)$ & 134.162 & $-\mathrm{DE} / \mathrm{DX}=$ & 0.0 & \\
\hline ! D23 & $\mathrm{D}(3,2,8,10)$ & -45.3109 & $-\mathrm{DE} / \mathrm{DX}=$ & 0.0 & \\
\hline ! D24 & $\mathrm{D}(7,2,8,9)$ & -115.574 & $-\mathrm{DE} / \mathrm{DX}=$ & 0.0 & \\
\hline ! D25 & $\mathrm{D}(7,2,8,10)$ & 64.9531 & $-\mathrm{DE} / \mathrm{DX}=$ & 0.0 & \\
\hline ! D26 & $\mathrm{D}(11,2,8,9)$ & 7.5242 & $-\mathrm{DE} / \mathrm{DX}=$ & 0.0 & \\
\hline ! D27 & $\mathrm{D}(11,2,8,10)$ & -171.9486 & $-\mathrm{DE} / \mathrm{DX}=$ & $=0.0$ & \\
\hline ! D28 & $\mathrm{D}(3,2,11,12)$ & -178.0061 & $-\mathrm{DE} / \mathrm{DX}=$ & $=0.0$ & \\
\hline ! D29 & $\mathrm{D}(3,2,11,13)$ & -59.2555 & $-\mathrm{DE} / \mathrm{DX}=$ & 0.0 & \\
\hline ! D30 & $\mathrm{D}(3,2,11,14)$ & 61.1066 & $-\mathrm{DE} / \mathrm{DX}=$ & 0.0 & \\
\hline ! D31 & $\mathrm{D}(7,2,11,12)$ & 58.5995 & $-\mathrm{DE} / \mathrm{DX}=$ & 0.0 & \\
\hline ! D32 & $\mathrm{D}(7,2,11,13)$ & 177.3501 & $-\mathrm{DE} / \mathrm{DX}=$ & 0.0 & \\
\hline ! D33 & $\mathrm{D}(7,2,11,14)$ & -62.2879 & $-\mathrm{DE} / \mathrm{DX}=$ & 0.0 & \\
\hline ! D34 & $\mathrm{D}(8,2,11,12)$ & -56.8622 & $-\mathrm{DE} / \mathrm{DX}=$ & 0.0 & \\
\hline ! D35 & $\mathrm{D}(8,2,11,13)$ & 61.8885 & $-\mathrm{DE} / \mathrm{DX}=$ & 0.0 & \\
\hline ! D36 & $\mathrm{D}(8,2,11,14)$ & -177.7495 & $-\mathrm{DE} / \mathrm{DX}=$ & $=0.0$ & \\
\hline ! D37 & $\mathrm{D}(2,3,4,1)$ & 3.7698 & $-\mathrm{DE} / \mathrm{DX}=0$ & 0.0 & \\
\hline ! D38 & $\mathrm{D}(2,3,4,6)$ & -177.0271 & $-\mathrm{DE} / \mathrm{DX}=$ & 0.0 & \\
\hline ! D39 & $\mathrm{D}(5,3,4,1)$ & -179.2234 & $-\mathrm{DE} / \mathrm{DX}=$ & 0.0 & \\
\hline ! D40 & $\mathrm{D}(5,3,4,6)$ & -0.0204 & $-\mathrm{DE} / \mathrm{DX}=$ & 0.0 & \\
\hline
\end{tabular}

GradGradGradGradGradGradGradGradGradGradGradGradGradGradGradGradGradGrad Input orientation: 


\begin{tabular}{|c|c|c|c|c|c|}
\hline \multirow{2}{*}{$\begin{array}{l}\text { Center } \\
\text { Number }\end{array}$} & Atomic & \multicolumn{2}{|c|}{ Atomic } & \multicolumn{2}{|c|}{ Coordinates (Angstrom } \\
\hline & Numbe & & Type & $\mathrm{X}$ & Z \\
\hline 1 & 6 & 0 & -0.030387 & -0.017473 & -0.012549 \\
\hline 2 & 6 & 0 & -0.027461 & 0.116025 & 2.568674 \\
\hline 3 & 6 & 0 & 1.315394 & 0.183591 & 1.913266 \\
\hline 4 & 6 & 0 & 1.297348 & 0.066948 & 0.568555 \\
\hline 5 & 1 & 0 & 2.219628 & 0.270388 & 2.506934 \\
\hline 6 & 1 & 0 & 2.192722 & 0.049559 & -0.045938 \\
\hline 7 & 15 & 0 & -1.292547 & 0.054277 & 1.142351 \\
\hline 8 & 6 & 0 & -0.138669 & -1.303940 & 3.155877 \\
\hline 9 & 8 & 0 & -0.533771 & -1.550929 & 4.267732 \\
\hline 10 & 1 & 0 & 0.189019 & -2.105292 & 2.464824 \\
\hline 11 & 6 & 0 & -0.302591 & 1.189128 & 3.625415 \\
\hline 12 & 1 & 0 & -1.285734 & 1.045591 & 4.075633 \\
\hline 13 & 1 & 0 & 0.436760 & 1.128621 & 4.428924 \\
\hline 14 & 1 & 0 & -0.258607 & 2.186406 & 3.183237 \\
\hline 15 & 6 & 0 & -0.220908 & -0.124892 & -1.497690 \\
\hline 16 & 1 & 0 & -1.275275 & -0.131242 & -1.778035 \\
\hline 17 & 1 & 0 & 0.266162 & 0.712075 & -2.010523 \\
\hline 18 & 1 & 0 & 0.237463 & -1.043870 & -1.879839 \\
\hline
\end{tabular}

Distance matrix (angstroms):

$\begin{array}{lllll}1 & 2 & 3 & 4 & 5\end{array}$

$1 \mathrm{C} \quad 0.000000$

2 C $2.584674 \quad 0.000000$

$\begin{array}{lllll}3 & \mathrm{C} & 2.358032 & 1.495788 & 0.000000\end{array}$

$\begin{array}{llllll}4 & \mathrm{C} & 1.451789 & 2.399584 & 1.349881 & 0.000000\end{array}$

$\begin{array}{lllllll}5 \mathrm{H} & 3.390166 & 2.253230 & 1.085180 & 2.156224 & 0.000000\end{array}$

$\begin{array}{lllllll}6 & \mathrm{H} & 2.224371 & 3.430717 & 2.150848 & 1.086093 & 2.562546\end{array}$ 


$$
\begin{aligned}
& \begin{array}{lllllll}
7 & \mathrm{P} & 1.712305 & 1.907525 & 2.722569 & 2.652727 & 3.774144
\end{array} \\
& \begin{array}{lllllll}
8 & \text { C } & 3.421351 & 1.540609 & 2.423042 & 3.261244 & 2.908814
\end{array} \\
& \begin{array}{lllllll}
9 & \mathrm{O} & 4.574460 & 2.433493 & 3.459983 & 4.433332 & 3.741499
\end{array} \\
& \begin{array}{lllllll}
10 & \mathrm{H} & 3.247230 & 2.234256 & 2.609966 & 3.089152 & 3.125541
\end{array} \\
& \begin{array}{lllllll}
11 & \mathrm{C} & 3.842495 & 1.530996 & 2.561335 & 3.628152 & 2.908036
\end{array} \\
& 12 \mathrm{H} \quad 4.406726 \quad 2.172158 \quad 3.490665 \quad 4.4642643 .917820 \\
& \begin{array}{lllllll}
13 & \mathrm{H} & 4.610688 & 2.168267 & 2.827299 & 4.095145 & 2.758483
\end{array}
\end{aligned}
$$

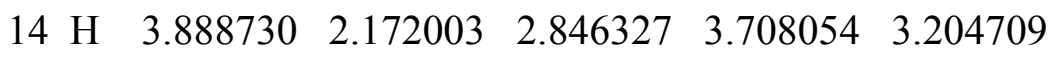

$$
\begin{aligned}
& \begin{array}{lllllll}
15 & \mathrm{C} & 1.501160 & 4.078085 & 3.753665 & 2.571239 & 4.706323
\end{array} \\
& \begin{array}{lllllll}
16 & \mathrm{H} & 2.163245 & 4.529024 & 4.520662 & 3.487715 & 5.544061
\end{array} \\
& \begin{array}{lllllll}
17 \mathrm{H} & 2.147577 & 4.627152 & 4.095888 & 2.851523 & 4.941512
\end{array} \\
& 18 \mathrm{H} \quad 2.147558 \quad 4.604867 \quad 4.129919 \quad 2.889966 \quad 4.989993 \\
& \begin{array}{lllll}
6 & 7 & 8 & 9 & 10
\end{array}
\end{aligned}
$$

$6 \mathrm{H} \quad 0.000000$

$\begin{array}{llll}7 \mathrm{P} & 3.682276 & 0.000000\end{array}$

$\begin{array}{lllll}8 & \mathrm{C} & 4.185566 & 2.688954 & 0.000000\end{array}$

$\begin{array}{llllll}9 & \mathrm{O} & 5.348184 & 3.594500 & 1.205541 & 0.000000\end{array}$

$10 \mathrm{H} \quad 3.868092 \quad 2.933890 \quad 1.107745 \quad 2.019955 \quad 0.000000$

$\begin{array}{lllllll}11 & \mathrm{C} & 4.583016 & 2.904050 & 2.542189 & 2.823815 & 3.527302\end{array}$

$\begin{array}{lllllll}12 & \mathrm{H} & 5.484440 & 3.096271 & 2.771643 & 2.710031 & 3.833754\end{array}$

$13 \mathrm{H} \quad 4.9266793 .866042 \quad 2.805195 \quad 2.854453 \quad 3.791735$

$14 \mathrm{H} \quad 4.582871 \quad 3.1273353 .492514 \quad 3.901221 \quad 4.374376$

$\begin{array}{lllllll}15 & \mathrm{C} & 2.821990 & 2.854878 & 4.801313 & 5.947399 & 4.448769\end{array}$

$\begin{array}{lllllll}16 & \mathrm{H} & 3.880703 & 2.926324 & 5.197171 & 6.254330 & 4.903355\end{array}$

$\begin{array}{lllllll}17 \mathrm{H} & 2.830223 & 3.578113 & 5.560567 & 6.721426 & 5.288880\end{array}$

$\begin{array}{lllllll}18 \mathrm{H} & 2.895137 & 3.560967 & 5.056436 & 6.216473 & 4.472702\end{array}$

$$
\begin{array}{lllll}
11 & 12 & 13 & 14 & 15
\end{array}
$$

$11 \mathrm{C} \quad 0.000000$

$12 \mathrm{H} \quad 1.0908110 .000000$

$13 \mathrm{H} \quad 1.093585 \quad 1.760310 \quad 0.000000$

$\begin{array}{llllll}14 & \mathrm{H} & 1.091797 & 1.775618 & 1.776002 & 0.000000\end{array}$ 


\begin{tabular}{|c|c|c|c|c|}
\hline C 5.28 & 5.793601 & 6.093322 & 594 & 00 \\
\hline 5.646837 & 5.970803 & 6.560843 & 5.569500 & 1.091019 \\
\hline 5.684616 & 6.289747 & 6.455161 & 5.424406 & 1.095787 \\
\hline 5.965380 & 6.492582 & 6.675322 & 6.026235 & 1.09572 \\
\hline
\end{tabular}

$16 \mathrm{H} \quad 0.000000$

$17 \mathrm{H} \quad 1.772360 \quad 0.000000$

$18 \mathrm{H} \quad 1.769641 \quad 1.761035 \quad 0.000000$

Stoichiometry C7H9OP

Zero-point correction=

Thermal correction to Energy=

Thermal correction to Enthalpy=

Thermal correction to Gibbs Free Energy=

Sum of electronic and zero-point Energies $=$

Sum of electronic and thermal Energies=

Sum of electronic and thermal Enthalpies=

Sum of electronic and thermal Free Energies=
0.141583 (Hartree/Particle)

0.151187

0.152131

0.106872

$-688.680769$

$-688.671165$

$-688.670221$

$-688.715480$

\section{3-Formyl-2,5-dimethyl-3H-phosphole}

! Optimized Parameters !

! (Angstroms and Degrees) !

! Name Definition

Value Derivative Info.

$\begin{array}{llcll}\text { ! R1 } & \mathrm{R}(1,2) & 1.3463 & -\mathrm{DE} / \mathrm{DX}=0.0 & ! \\ \text { ! R2 } & \mathrm{R}(1,4) & 1.8432 & -\mathrm{DE} / \mathrm{DX}=0.0 & ! \\ \text { ! R3 } & \mathrm{R}(1,5) & 1.5011 & -\mathrm{DE} / \mathrm{DX}=0.0 & ! \\ \text { ! R4 } & \mathrm{R}(2,3) & 1.0861 & -\mathrm{DE} / \mathrm{DX}=0.0 & ! \\ \text { ! R5 } & \mathrm{R}(2,14) & 1.4974 & -\mathrm{DE} / \mathrm{DX}=0.0 & ! \\ \text { ! R6 } & \mathrm{R}(4,9) & 1.6979 & -\mathrm{DE} / \mathrm{DX}=0.0 & ! \\ \text { ! R7 } & \mathrm{R}(5,6) & 1.0947 & -\mathrm{DE} / \mathrm{DX}=0.0 & !\end{array}$




\begin{tabular}{|c|c|c|c|c|c|}
\hline ! R8 & $\mathrm{R}(5,7)$ & 1.0948 & $-\mathrm{DE} / \mathrm{DX}=0.0$ & .0 & $!$ \\
\hline ! R9 & $\mathrm{R}(5,8)$ & 1.0927 & $-\mathrm{DE} / \mathrm{DX}=0.0$ & .0 & $!$ \\
\hline ! R10 & $\mathrm{R}(9,10)$ & 1.4996 & $-\mathrm{DE} / \mathrm{DX}=0$ & 0.0 & $!$ \\
\hline ! R11 & $\mathrm{R}(9,14)$ & 1.5088 & $-\mathrm{DE} / \mathrm{DX}=0$ & 0.0 & $!$ \\
\hline ! R12 & $\mathrm{R}(10,11)$ & 1.0914 & $-\mathrm{DE} / \mathrm{DX}=0$ & 0.0 & $!$ \\
\hline ! R13 & $\mathrm{R}(10,12)$ & 1.0957 & $-\mathrm{DE} / \mathrm{DX}=0$ & 0.0 & $!$ \\
\hline ! R14 & $\mathrm{R}(10,13)$ & 1.0965 & $-\mathrm{DE} / \mathrm{DX}=0$ & 0.0 & $!$ \\
\hline ! R15 & $\mathrm{R}(14,15)$ & 1.0945 & $-\mathrm{DE} / \mathrm{DX}=0$ & 0.0 & $!$ \\
\hline ! R16 & $\mathrm{R}(14,16)$ & 1.5421 & $-\mathrm{DE} / \mathrm{DX}=0$ & 0.0 & $!$ \\
\hline ! R17 & $\mathrm{R}(16,17)$ & 1.1084 & $-\mathrm{DE} / \mathrm{DX}=0$ & 0.0 & $!$ \\
\hline ! R18 & $\mathrm{R}(16,18)$ & 1.2031 & $-\mathrm{DE} / \mathrm{DX}=0$ & 0.0 & $!$ \\
\hline ! A1 & $\mathrm{A}(2,1,4)$ & 111.3843 & $-\mathrm{DE} / \mathrm{DX}=$ & 0.0 & $!$ \\
\hline ! A2 & $\mathrm{A}(2,1,5)$ & 125.9947 & $-\mathrm{DE} / \mathrm{DX}=$ & 0.0 & $!$ \\
\hline ! A3 & $\mathrm{A}(4,1,5)$ & 122.6119 & $-\mathrm{DE} / \mathrm{DX}=$ & 0.0 & $!$ \\
\hline ! A4 & $\mathrm{A}(1,2,3)$ & 124.1243 & $-\mathrm{DE} / \mathrm{DX}=$ & 0.0 & $!$ \\
\hline ! A5 & $\mathrm{A}(1,2,14)$ & 115.0516 & $-\mathrm{DE} / \mathrm{DX}=$ & 0.0 & $!$ \\
\hline ! A6 & $\mathrm{A}(3,2,14)$ & 120.7261 & $-\mathrm{DE} / \mathrm{DX}=$ & 0.0 & $!$ \\
\hline ! A7 & $\mathrm{A}(1,4,9)$ & 91.975 & $-\mathrm{DE} / \mathrm{DX}=0$ & .0 & $!$ \\
\hline ! A8 & $\mathrm{A}(1,5,6)$ & 111.1617 & $-\mathrm{DE} / \mathrm{DX}=$ & 0.0 & $!$ \\
\hline ! A9 & $\mathrm{A}(1,5,7)$ & 111.1446 & $-\mathrm{DE} / \mathrm{DX}=$ & 0.0 & $!$ \\
\hline ! A10 & $\mathrm{A}(1,5,8)$ & 111.3042 & $-\mathrm{DE} / \mathrm{DX}=$ & 0.0 & $!$ \\
\hline ! A11 & $\mathrm{A}(6,5,7)$ & 106.7764 & $-\mathrm{DE} / \mathrm{DX}=$ & 0.0 & $!$ \\
\hline ! A12 & $\mathrm{A}(6,5,8)$ & 108.0956 & $-\mathrm{DE} / \mathrm{DX}=$ & 0.0 & $!$ \\
\hline ! A13 & $\mathrm{A}(7,5,8)$ & 108.176 & $-\mathrm{DE} / \mathrm{DX}=$ & 0.0 & $!$ \\
\hline ! A14 & $\mathrm{A}(4,9,10)$ & 126.3136 & $-\mathrm{DE} / \mathrm{DX}=$ & 0.0 & ! \\
\hline ! A15 & $\mathrm{A}(4,9,14)$ & 113.1497 & $-\mathrm{DE} / \mathrm{DX}=$ & 0.0 & $!$ \\
\hline ! A16 & $\mathrm{A}(10,9,14)$ & 120.5277 & $-\mathrm{DE} / \mathrm{DX}=$ & 0.0 & ! \\
\hline ! A17 & $\mathrm{A}(9,10,11)$ & 111.6804 & $-\mathrm{DE} / \mathrm{DX}=$ & 0.0 & ! \\
\hline ! A18 & $\mathrm{A}(9,10,12)$ & 111.7415 & $-\mathrm{DE} / \mathrm{DX}=$ & 0.0 & ! \\
\hline ! A19 & $\mathrm{A}(9,10,13)$ & 110.6169 & $-\mathrm{DE} / \mathrm{DX}=$ & 0.0 & ! \\
\hline A20 & $\mathrm{A}(11,10,12)$ & 108.1512 & $-\mathrm{DE} / \mathrm{DX}=$ & $=0.0$ & \\
\hline
\end{tabular}




\begin{tabular}{|c|c|c|c|c|c|}
\hline ! A21 & $\mathrm{A}(11,10,13)$ & 107.9226 & $-\mathrm{DE} / \mathrm{DX}=$ & 0.0 & ! \\
\hline ! A22 & $\mathrm{A}(12,10,13)$ & 106.5073 & $-\mathrm{DE} / \mathrm{DX}=$ & 0.0 & $!$ \\
\hline ! A23 & $\mathrm{A}(2,14,9)$ & 108.0803 & $-\mathrm{DE} / \mathrm{DX}=$ & 0.0 & $!$ \\
\hline ! A24 & $\mathrm{A}(2,14,15)$ & 114.1563 & $-\mathrm{DE} / \mathrm{DX}=$ & 0.0 & 1 \\
\hline ! A25 & $\mathrm{A}(2,14,16)$ & 107.8314 & -DE/DX = & 0.0 & 1 \\
\hline ! A26 & $\mathrm{A}(9,14,15)$ & 112.99 & $-\mathrm{DE} / \mathrm{DX}=$ & 0.0 & $!$ \\
\hline ! A27 & $\mathrm{A}(9,14,16)$ & 106.0623 & -DE/DX = & 0.0 & $!$ \\
\hline ! A28 & $\mathrm{A}(15,14,16)$ & 107.2948 & $-\mathrm{DE} / \mathrm{DX}=$ & 0.0 & $!$ \\
\hline ! A29 & $\mathrm{A}(14,16,17)$ & 113.3537 & $-\mathrm{DE} / \mathrm{DX}=$ & 0.0 & $!$ \\
\hline ! A30 & $\mathrm{A}(14,16,18)$ & 124.6033 & $-\mathrm{DE} / \mathrm{DX}=$ & 0.0 & ! \\
\hline ! A31 & $\mathrm{A}(17,16,18)$ & 122.0358 & $-\mathrm{DE} / \mathrm{DX}=$ & 0.0 & $!$ \\
\hline ! D1 & $\mathrm{D}(4,1,2,3)$ & 179.9198 & $-\mathrm{DE} / \mathrm{DX}=$ & 0.0 & $!$ \\
\hline ! D2 & $\mathrm{D}(4,1,2,14)$ & 3.5073 & $-\mathrm{DE} / \mathrm{DX}=0$ & 0.0 & $!$ \\
\hline ! D3 & $\mathrm{D}(5,1,2,3)$ & -1.1581 & $-\mathrm{DE} / \mathrm{DX}=0$ & 0.0 & $!$ \\
\hline ! D4 & $\mathrm{D}(5,1,2,14)$ & -177.5706 & $-\mathrm{DE} / \mathrm{DX}=$ & 0.0 & $!$ \\
\hline ! D5 & $\mathrm{D}(2,1,4,9)$ & 0.2169 & $-\mathrm{DE} / \mathrm{DX}=0$ & 0.0 & $!$ \\
\hline ! D6 & $\mathrm{D}(5,1,4,9)$ & -178.7477 & $-\mathrm{DE} / \mathrm{DX}=$ & 0.0 & $!$ \\
\hline ! D7 & $\mathrm{D}(2,1,5,6)$ & 120.2857 & $-\mathrm{DE} / \mathrm{DX}=$ & 0.0 & $!$ \\
\hline ! D8 & $\mathrm{D}(2,1,5,7)$ & -120.9263 & $-\mathrm{DE} / \mathrm{DX}=$ & 0.0 & $!$ \\
\hline ! D9 & $\mathrm{D}(2,1,5,8)$ & -0.2751 & $-\mathrm{DE} / \mathrm{DX}=0$ & 0.0 & $!$ \\
\hline ! D10 & $\mathrm{D}(4,1,5,6)$ & -60.9059 & $-\mathrm{DE} / \mathrm{DX}=$ & 0.0 & $!$ \\
\hline ! D11 & $\mathrm{D}(4,1,5,7)$ & 57.8821 & $-\mathrm{DE} / \mathrm{DX}=$ & 0.0 & $!$ \\
\hline ! D12 & $\mathrm{D}(4,1,5,8)$ & 178.5333 & $-\mathrm{DE} / \mathrm{DX}=$ & 0.0 & $!$ \\
\hline ! D13 & $\mathrm{D}(1,2,14,9)$ & -6.1072 & $-\mathrm{DE} / \mathrm{DX}=$ & 0.0 & $!$ \\
\hline ! D14 & $\mathrm{D}(1,2,14,15)$ & -132.7419 & $-\mathrm{DE} / \mathrm{DX}=$ & $=0.0$ & ! \\
\hline ! D15 & $\mathrm{D}(1,2,14,16)$ & 108.1452 & $-\mathrm{DE} / \mathrm{DX}=$ & 0.0 & ! \\
\hline ! D16 & $\mathrm{D}(3,2,14,9)$ & 177.3474 & -DE/DX = & 0.0 & $!$ \\
\hline ! D17 & $\mathrm{D}(3,2,14,15)$ & 50.7127 & $-\mathrm{DE} / \mathrm{DX}=$ & 0.0 & $!$ \\
\hline ! D18 & $\mathrm{D}(3,2,14,16)$ & -68.4001 & $-\mathrm{DE} / \mathrm{DX}=$ & 0.0 & ! \\
\hline D19 & $\mathrm{D}(1,4,9,10)$ & 177.299 & $-\mathrm{DE} / \mathrm{DX}=$ & 0.0 & $!$ \\
\hline 20 & $\mathrm{D}(1,4,9,14)$ & -3.7957 & $-\mathrm{DE} / \mathrm{DX}=$ & 0.0 & $!$ \\
\hline
\end{tabular}




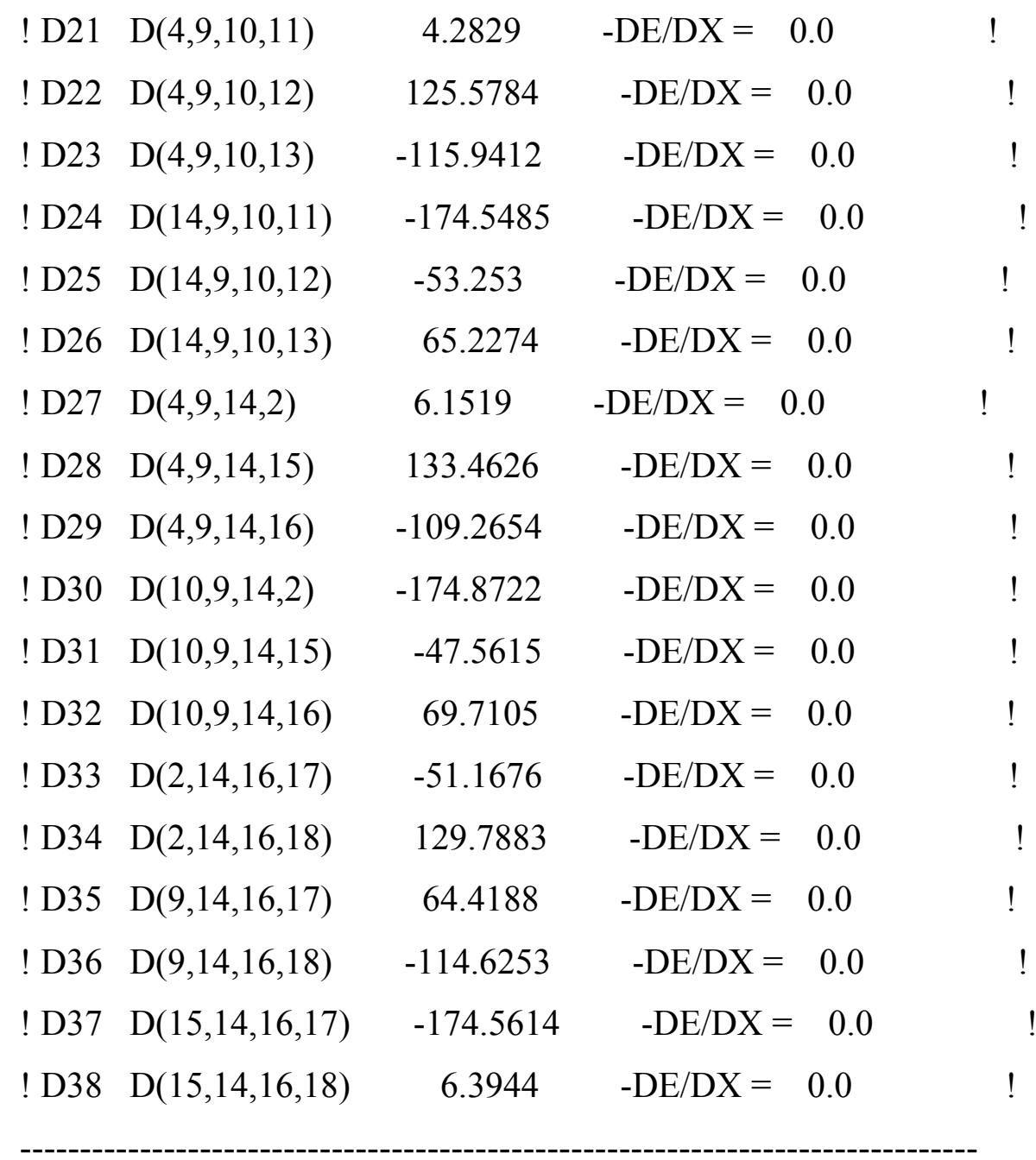

GradGradGradGradGradGradGradGradGradGradGradGradGradGradGradGradGradGrad

Input orientation:

\begin{tabular}{cccccc} 
Center & \multicolumn{2}{c}{ Atomic } & \multicolumn{2}{c}{ Atomic } & \multicolumn{2}{c}{ Coordinates (Angstroms) } \\
Number & Number & Type & X & Y & Z \\
& & & & & \\
1 & 6 & 0 & -0.016896 & -0.031274 & 0.034576 \\
2 & 6 & 0 & -0.013378 & 0.167005 & 1.366216 \\
3 & 1 & 0 & 0.886471 & 0.293881 & 1.960931 \\
4 & 15 & 0 & -1.733341 & -0.203543 & -0.614728
\end{tabular}




\begin{tabular}{|c|c|c|c|c|c|}
\hline 5 & 6 & 0 & 1.192917 & -0.086773 & -0.852289 \\
\hline 6 & 1 & 0 & 1.263309 & -1.051548 & -1.364784 \\
\hline 7 & 1 & 0 & 1.147672 & 0.681889 & -1.630532 \\
\hline 8 & 1 & 0 & 2.111896 & 0.061348 & -0.279912 \\
\hline 9 & 6 & 0 & -2.402121 & 0.003623 & 0.932131 \\
\hline 10 & 6 & 0 & -3.862575 & 0.050264 & 1.269249 \\
\hline 11 & 1 & 0 & -4.484883 & -0.142297 & 0.393521 \\
\hline 12 & 1 & 0 & -4.124404 & -0.681488 & 2.041639 \\
\hline 13 & 1 & 0 & -4.135677 & 1.034770 & 1.667394 \\
\hline 14 & 6 & 0 & -1.360851 & 0.120561 & 2.017754 \\
\hline 15 & 1 & 0 & -1.553587 & 0.948198 & 2.707604 \\
\hline 16 & 6 & 0 & -1.446254 & -1.185389 & 2.833314 \\
\hline 17 & 1 & 0 & -1.171974 & -2.086768 & 2.249455 \\
\hline 18 & 8 & 0 & -1.790403 & -1.250862 & 3.984252 \\
\hline
\end{tabular}

Distance matrix (angstroms):

$\begin{array}{lllll}1 & 2 & 3 & 4 & 5\end{array}$

$1 \mathrm{C} \quad 0.000000$

2 C 1.3463250 .000000

$\begin{array}{llll}3 \mathrm{H} & 2.152357 & 1.086053 & 0.000000\end{array}$

$\begin{array}{llllll}4 & \mathrm{P} & 1.843219 & 2.649475 & 3.707406 & 0.000000\end{array}$

$\begin{array}{lllllll}5 & \mathrm{C} & 1.501085 & 2.537974 & 2.855348 & 2.938206 & 0.000000\end{array}$

$\begin{array}{lllllll}6 & \mathrm{H} & 2.153623 & 3.251640 & 3.607294 & 3.203374 & 1.094714\end{array}$

$\begin{array}{lllllll}7 & \mathrm{H} & 2.153464 & 3.254787 & 3.621793 & 3.180579 & 1.094783\end{array}$

$\begin{array}{lllllll}8 & \mathrm{H} & 2.153889 & 2.690296 & 2.564589 & 3.868865 & 1.092738\end{array}$

$\begin{array}{lllllll}9 & \mathrm{C} & 2.548750 & 2.433356 & 3.457964 & 1.697927 & 4.014552\end{array}$

$\begin{array}{lllllll}10 \mathrm{C} & 4.039840 & 3.852187 & 4.805331 & 2.854369 & 5.484314\end{array}$

$11 \mathrm{H} \quad 4.483757 \quad 4.586519 \quad 5.612349 \quad 2.931092 \quad 5.813135$

$\begin{array}{lllllll}12 & \mathrm{H} & 4.617651 & 4.251667 & 5.105558 & 3.605815 & 6.082961\end{array}$

$\begin{array}{lllllll}13 & \mathrm{H} & 4.557072 & 4.223397 & 5.084983 & 3.537333 & 6.000049\end{array}$

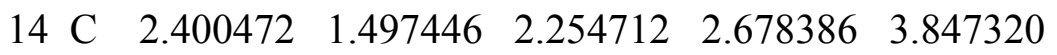




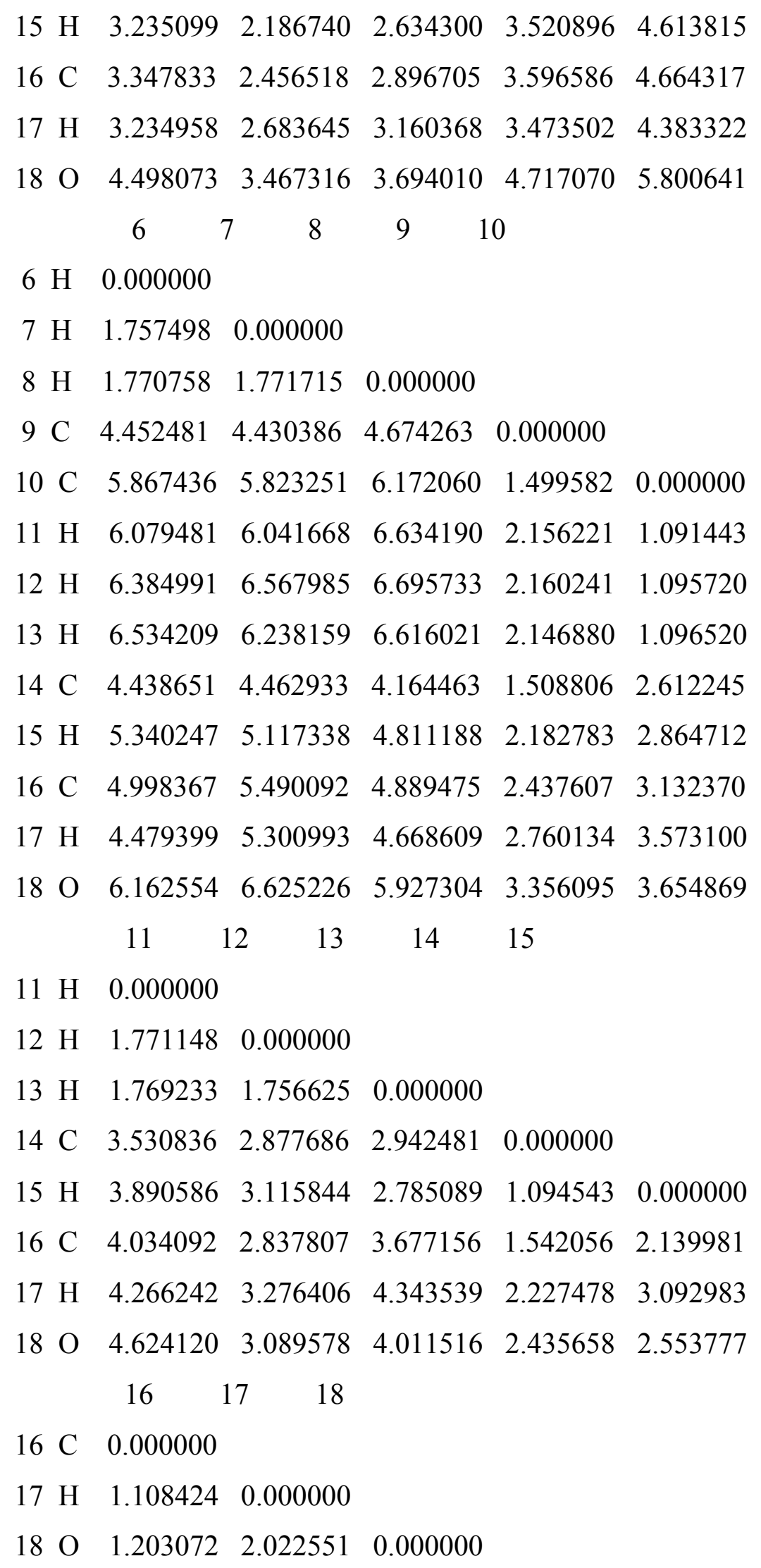


Stoichiometry C7H9OP

Zero-point correction $=$

0.141279 (Hartree/Particle)

Thermal correction to Energy=

0.151126

Thermal correction to Enthalpy=

0.152070

Thermal correction to Gibbs Free Energy=

0.105730

Sum of electronic and zero-point Energies= $-688.678112$

Sum of electronic and thermal Energies $=$ $-688.668265$

Sum of electronic and thermal Enthalpies $=$ $-688.667321$

Sum of electronic and thermal Free Energies= $-688.713661$

\section{TS 1H-2H-phosphole}

Optimized Parameters !

! (Angstroms and Degrees) !

! Name Definition Value Derivative Info.

\begin{tabular}{|c|c|c|c|c|}
\hline ! R1 & $\mathrm{R}(1,4)$ & 1.3943 & $-\mathrm{DE} / \mathrm{DX}=$ & 0.0 \\
\hline ! R2 & $\mathrm{R}(1,7)$ & 1.7732 & $-\mathrm{DE} / \mathrm{DX}=$ & 0.0 \\
\hline ! R3 & $\mathrm{R}(1,15)$ & 1.505 & $-\mathrm{DE} / \mathrm{DX}=$ & 0.0 \\
\hline ! R4 & $\mathrm{R}(2,3)$ & 1.4065 & $-\mathrm{DE} / \mathrm{DX}=$ & 0.0 \\
\hline ! R5 & $\mathrm{R}(2,7)$ & 1.8279 & $-\mathrm{DE} / \mathrm{DX}=$ & 0.0 \\
\hline ! R6 & $\mathrm{R}(2,8)$ & 1.9124 & $-\mathrm{DE} / \mathrm{DX}=$ & 0.0 \\
\hline ! R7 & $\mathrm{R}(2,11)$ & 1.5042 & $-\mathrm{DE} / \mathrm{DX}=$ & 0.0 \\
\hline ! R8 & $\mathrm{R}(3,4)$ & 1.4033 & $-\mathrm{DE} / \mathrm{DX}=$ & 0.0 \\
\hline ! R9 & $\mathrm{R}(3,5)$ & 1.0851 & $-\mathrm{DE} / \mathrm{DX}=$ & 0.0 \\
\hline ! R10 & $\mathrm{R}(4,6)$ & 1.0861 & $-\mathrm{DE} / \mathrm{DX}=$ & 0.0 \\
\hline ! R11 & $\mathrm{R}(7,8)$ & 2.1798 & $-\mathrm{DE} / \mathrm{DX}=$ & 0.0 \\
\hline ! R12 & $\mathrm{R}(8,9)$ & 1.1907 & $-\mathrm{DE} / \mathrm{DX}=$ & 0.0 \\
\hline ! R13 & $\mathrm{R}(8,10)$ & 1.1034 & $-\mathrm{DE} / \mathrm{DX}=$ & 0.0 \\
\hline ! R14 & $\mathrm{R}(11,12)$ & 1.0924 & $-\mathrm{DE} / \mathrm{DX}=$ & 0.0 \\
\hline $\mathrm{R} 15$ & $\mathrm{R}(11,13)$ & 1.0936 & $-\mathrm{DE} / \mathrm{DX}=$ & 0.0 \\
\hline
\end{tabular}




\begin{tabular}{|c|c|c|c|c|c|}
\hline ! R16 & $\mathrm{R}(11,14)$ & 1.0934 & $-\mathrm{DE} / \mathrm{DX}=$ & 0.0 & $!$ \\
\hline ! R17 & $\mathrm{R}(15,16)$ & 1.0923 & $-\mathrm{DE} / \mathrm{DX}=$ & 0.0 & $!$ \\
\hline ! R18 & $\mathrm{R}(15,17)$ & 1.0957 & $-\mathrm{DE} / \mathrm{DX}=$ & 0.0 & $!$ \\
\hline ! R19 & $\mathrm{R}(15,18)$ & 1.0935 & $-\mathrm{DE} / \mathrm{DX}=$ & 0.0 & $!$ \\
\hline ! A1 & $\mathrm{A}(4,1,7)$ & 111.4397 & $-\mathrm{DE} / \mathrm{DX}=$ & 0.0 & $!$ \\
\hline ! A2 & $\mathrm{A}(4,1,15)$ & 124.3238 & $-\mathrm{DE} / \mathrm{DX}=$ & 0.0 & $!$ \\
\hline ! A3 & $\mathrm{A}(7,1,15)$ & 124.2202 & $-\mathrm{DE} / \mathrm{DX}=$ & 0.0 & $!$ \\
\hline ! A4 & $\mathrm{A}(3,2,7)$ & 109.6798 & $-\mathrm{DE} / \mathrm{DX}=$ & 0.0 & $!$ \\
\hline ! A5 & $\mathrm{A}(3,2,8)$ & 99.656 & $-\mathrm{DE} / \mathrm{DX}=0$ & .0 & $!$ \\
\hline ! A6 & $\mathrm{A}(3,2,11)$ & 124.8254 & $-\mathrm{DE} / \mathrm{DX}=$ & 0.0 & $!$ \\
\hline ! A7 & $\mathrm{A}(7,2,11)$ & 124.0597 & $-\mathrm{DE} / \mathrm{DX}=$ & 0.0 & $!$ \\
\hline ! A8 & $\mathrm{A}(8,2,11)$ & 107.8621 & $-\mathrm{DE} / \mathrm{DX}=$ & 0.0 & $!$ \\
\hline ! A9 & $\mathrm{A}(2,3,4)$ & 113.7712 & $-\mathrm{DE} / \mathrm{DX}=$ & 0.0 & $!$ \\
\hline ! A10 & $\mathrm{A}(2,3,5)$ & 122.7017 & $-\mathrm{DE} / \mathrm{DX}=$ & 0.0 & $!$ \\
\hline ! A11 & $\mathrm{A}(4,3,5)$ & 123.4743 & $-\mathrm{DE} / \mathrm{DX}=$ & 0.0 & $!$ \\
\hline ! A12 & $\mathrm{A}(1,4,3)$ & 114.6957 & $-\mathrm{DE} / \mathrm{DX}=$ & 0.0 & $!$ \\
\hline ! A13 & $\mathrm{A}(1,4,6)$ & 122.7104 & $-\mathrm{DE} / \mathrm{DX}=$ & 0.0 & $!$ \\
\hline ! A14 & $\mathrm{A}(3,4,6)$ & 122.5596 & $-\mathrm{DE} / \mathrm{DX}=$ & 0.0 & $!$ \\
\hline ! A15 & $\mathrm{A}(1,7,2)$ & 90.3047 & $-\mathrm{DE} / \mathrm{DX}=$ & 0.0 & $!$ \\
\hline ! A16 & $\mathrm{A}(1,7,8)$ & 90.9175 & $-\mathrm{DE} / \mathrm{DX}=$ & 0.0 & $!$ \\
\hline ! A17 & $\mathrm{A}(2,8,9)$ & 119.1253 & $-\mathrm{DE} / \mathrm{DX}=$ & 0.0 & $!$ \\
\hline ! A18 & $\mathrm{A}(2,8,10)$ & 105.4089 & $-\mathrm{DE} / \mathrm{DX}=$ & 0.0 & $!$ \\
\hline ! A19 & $\mathrm{A}(7,8,9)$ & 124.0885 & $-\mathrm{DE} / \mathrm{DX}=$ & 0.0 & $!$ \\
\hline ! A20 & $\mathrm{A}(7,8,10)$ & 102.8716 & $-\mathrm{DE} / \mathrm{DX}=$ & 0.0 & $!$ \\
\hline ! A21 & $\mathrm{A}(9,8,10)$ & 128.3844 & $-\mathrm{DE} / \mathrm{DX}=$ & 0.0 & $!$ \\
\hline ! A22 & $\mathrm{A}(2,11,12)$ & 111.1175 & $-\mathrm{DE} / \mathrm{DX}=$ & 0.0 & $!$ \\
\hline ! A23 & $\mathrm{A}(2,11,13)$ & 110.9685 & $-\mathrm{DE} / \mathrm{DX}=$ & 0.0 & $!$ \\
\hline ! A24 & $\mathrm{A}(2,11,14)$ & 110.4186 & $-\mathrm{DE} / \mathrm{DX}=$ & 0.0 & $!$ \\
\hline ! A25 & $\mathrm{A}(12,11,13)$ & 107.8827 & $-\mathrm{DE} / \mathrm{DX}=$ & 0.0 & ! \\
\hline ! A26 & $\mathrm{A}(12,11,14)$ & 107.9995 & $-\mathrm{DE} / \mathrm{DX}=$ & 0.0 & ! \\
\hline $\mathrm{A} 27$ & $\mathrm{~A}(13,11,14)$ & 108.3399 & $-\mathrm{DE} / \mathrm{DX}=$ & 0.0 & ! \\
\hline
\end{tabular}




\begin{tabular}{|c|c|c|c|c|}
\hline ! A28 & $\mathrm{A}(1,15,16)$ & 111.8776 & $-\mathrm{DE} / \mathrm{DX}=0.0$ & $!$ \\
\hline ! A29 & $\mathrm{A}(1,15,17)$ & 110.6952 & $-\mathrm{DE} / \mathrm{DX}=0.0$ & $!$ \\
\hline ! A30 & $\mathrm{A}(1,15,18)$ & 111.4595 & $-\mathrm{DE} / \mathrm{DX}=0.0$ & $!$ \\
\hline ! A31 & $\mathrm{A}(16,15,17)$ & 107.1383 & $-\mathrm{DE} / \mathrm{DX}=0.0$ & $!$ \\
\hline ! A32 & $\mathrm{A}(16,15,18)$ & 108.0936 & $-\mathrm{DE} / \mathrm{DX}=0.0$ & , \\
\hline ! A33 & $\mathrm{A}(17,15,18)$ & 107.362 & $-\mathrm{DE} / \mathrm{DX}=0.0$ & $!$ \\
\hline ! D1 & $\mathrm{D}(7,1,4,3)$ & 0.0476 & $-\mathrm{DE} / \mathrm{DX}=0.0$ & $!$ \\
\hline ! D2 & $\mathrm{D}(7,1,4,6)$ & 177.966 & $-\mathrm{DE} / \mathrm{DX}=0.0$ & $!$ \\
\hline ! D3 & $\mathrm{D}(15,1,4,3)$ & -178.5343 & $-\mathrm{DE} / \mathrm{DX}=0.0$ & $!$ \\
\hline ! D4 & $\mathrm{D}(15,1,4,6)$ & -0.6159 & $-\mathrm{DE} / \mathrm{DX}=0.0$ & $!$ \\
\hline ! D5 & $\mathrm{D}(4,1,7,2)$ & -1.7042 & $-\mathrm{DE} / \mathrm{DX}=0.0$ & $!$ \\
\hline ! D6 & $\mathrm{D}(4,1,7,8)$ & -57.8819 & $-\mathrm{DE} / \mathrm{DX}=0.0$ & $!$ \\
\hline ! D7 & $\mathrm{D}(15,1,7,2)$ & 176.8794 & $-\mathrm{DE} / \mathrm{DX}=0.0$ & $!$ \\
\hline ! D8 & $\mathrm{D}(15,1,7,8)$ & 120.7017 & $-\mathrm{DE} / \mathrm{DX}=0.0$ & $!$ \\
\hline ! D9 & $\mathrm{D}(4,1,15,16)$ & 155.631 & $-\mathrm{DE} / \mathrm{DX}=0.0$ & $!$ \\
\hline ! D10 & $\mathrm{D}(4,1,15,17)$ & -84.9531 & $-\mathrm{DE} / \mathrm{DX}=0.0$ & $!$ \\
\hline ! D11 & $\mathrm{D}(4,1,15,18)$ & 34.4699 & $-\mathrm{DE} / \mathrm{DX}=0.0$ & $!$ \\
\hline ! D12 & $\mathrm{D}(7,1,15,16)$ & -22.7726 & $-\mathrm{DE} / \mathrm{DX}=0.0$ & $!$ \\
\hline ! D13 & $\mathrm{D}(7,1,15,17)$ & 96.6433 & $-\mathrm{DE} / \mathrm{DX}=0.0$ & $!$ \\
\hline ! D14 & $\mathrm{D}(7,1,15,18)$ & -143.9337 & $-\mathrm{DE} / \mathrm{DX}=0.0$ & $!$ \\
\hline ! D15 & $\mathrm{D}(7,2,3,4)$ & -3.4988 & $-\mathrm{DE} / \mathrm{DX}=0.0$ & $!$ \\
\hline ! D16 & $\mathrm{D}(7,2,3,5)$ & 179.0611 & $-\mathrm{DE} / \mathrm{DX}=0.0$ & $!$ \\
\hline ! D17 & $\mathrm{D}(8,2,3,4)$ & 69.9228 & $-\mathrm{DE} / \mathrm{DX}=0.0$ & $!$ \\
\hline ! D18 & $\mathrm{D}(8,2,3,5)$ & -107.5173 & $-\mathrm{DE} / \mathrm{DX}=0.0$ & $!$ \\
\hline ! D19 & $\mathrm{D}(11,2,3,4)$ & -170.2501 & $-\mathrm{DE} / \mathrm{DX}=0.0$ & $!$ \\
\hline ! D20 & $\mathrm{D}(11,2,3,5)$ & 12.3098 & $-\mathrm{DE} / \mathrm{DX}=0.0$ & $!$ \\
\hline ! D21 & $\mathrm{D}(3,2,7,1)$ & 2.927 & $-\mathrm{DE} / \mathrm{DX}=0.0$ & $!$ \\
\hline ! D22 & $\mathrm{D}(11,2,7,1)$ & 169.8014 & $-\mathrm{DE} / \mathrm{DX}=0.0$ & $!$ \\
\hline ! D23 & $\mathrm{D}(3,2,8,9)$ & 139.936 & $-\mathrm{DE} / \mathrm{DX}=0.0$ & $!$ \\
\hline ! D24 & $\mathrm{D}(3,2,8,10)$ & -13.0477 & $-\mathrm{DE} / \mathrm{DX}=0.0$ & $!$ \\
\hline 25 & $\mathrm{D}(11,2,8,9)$ & 8.3722 & $-\mathrm{DE} / \mathrm{DX}=0.0$ & $!$ \\
\hline
\end{tabular}




\begin{tabular}{|c|c|c|c|c|c|}
\hline ! D26 & $\mathrm{D}(11,2,8,10)$ & -144.6115 & $-\mathrm{DE} / \mathrm{DX}=$ & $=0.0$ & \\
\hline ! D27 & $\mathrm{D}(3,2,11,12)$ & -149.8515 & $-\mathrm{DE} / \mathrm{DX}=$ & $=0.0$ & \\
\hline ! D28 & $\mathrm{D}(3,2,11,13)$ & -29.8162 & $-\mathrm{DE} / \mathrm{DX}=$ & 0.0 & \\
\hline ! D29 & $\mathrm{D}(3,2,11,14)$ & 90.3311 & $-\mathrm{DE} / \mathrm{DX}=$ & 0.0 & \\
\hline ! D30 & $\mathrm{D}(7,2,11,12)$ & 45.2468 & $-\mathrm{DE} / \mathrm{DX}=$ & 0.0 & \\
\hline ! D31 & $\mathrm{D}(7,2,11,13)$ & 165.282 & $-\mathrm{DE} / \mathrm{DX}=$ & 0.0 & \\
\hline ! D32 & $\mathrm{D}(7,2,11,14)$ & -74.5707 & $-\mathrm{DE} / \mathrm{DX}=$ & 0.0 & \\
\hline ! D33 & $\mathrm{D}(8,2,11,12)$ & -33.8199 & $-\mathrm{DE} / \mathrm{DX}=$ & 0.0 & \\
\hline ! D34 & $\mathrm{D}(8,2,11,13)$ & 86.2154 & $-\mathrm{DE} / \mathrm{DX}=$ & 0.0 & \\
\hline ! D35 & $\mathrm{D}(8,2,11,14)$ & -153.6373 & $-\mathrm{DE} / \mathrm{DX}=$ & $=0.0$ & \\
\hline ! D36 & $\mathrm{D}(2,3,4,1)$ & 2.3344 & $-\mathrm{DE} / \mathrm{DX}=$ & 0.0 & $!$ \\
\hline ! D37 & $\mathrm{D}(2,3,4,6)$ & -175.5876 & $-\mathrm{DE} / \mathrm{DX}=$ & 0.0 & ! \\
\hline ! D38 & $\mathrm{D}(5,3,4,1)$ & 179.7518 & $-\mathrm{DE} / \mathrm{DX}=$ & 0.0 & ! \\
\hline ! D39 & $\mathrm{D}(5,3,4,6)$ & 1.8299 & $-\mathrm{DE} / \mathrm{DX}=$ & 0.0 & $!$ \\
\hline ! D40 & $\mathrm{D}(1,7,8,9)$ & -167.4167 & $-\mathrm{DE} / \mathrm{DX}=$ & 0.0 & ! \\
\hline D41 & $\mathrm{D}(1,7,8,10)$ & -9.9383 & $-\mathrm{DE} / \mathrm{DX}=$ & 0.0 & ! \\
\hline
\end{tabular}

GradGradGradGradGradGradGradGradGradGradGradGradGradGradGradGradGradGrad

Final structure in terms of initial Z-matrix:

C

C, 1,B1

$\mathrm{C}, 2, \mathrm{~B} 2,1, \mathrm{~A} 1$

C, 1,B3,3,A2,2,D1,0

H,3,B4,2,A3, 1,D2,0

$\mathrm{H}, 4, \mathrm{~B} 5,1, \mathrm{~A} 4,3, \mathrm{D} 3,0$

$\mathrm{P}, 1, \mathrm{~B} 6,4, \mathrm{~A} 5,3, \mathrm{D} 4,0$

C,2,B7,1,A6,4,D5,0

$\mathrm{O}, 8, \mathrm{~B} 8,2, \mathrm{~A} 7,1, \mathrm{D} 6,0$

H,8,B9,2,A8, 1,D7,0 
C,2,B10,1,A9,4,D8,0

H,11,B11,2,A10,1,D9,0

H,11,B12,2,A11,1,D10,0

H,11,B13,2,A12,1,D11,0

C,1,B14,4,A13,3,D12,0

H,15,B15,1,A14,4,D13,0

H,15,B16,1,A15,4,D14,0

H,15,B17,1,A16,4,D15,0

Variables:

$\mathrm{B} 1=2.55336125$

$\mathrm{B} 2=1.40649862$

B3=1.39425621

B4=1.08507668

B5=1.08610903

B6=1.77315041

B7=1.91235908

$\mathrm{B} 8=1.19067568$

B9=1.10338791

$\mathrm{B} 10=1.50419713$

B11=1.0923909

$\mathrm{B} 12=1.0936336$

$\mathrm{B} 13=1.09340588$

B14=1.50499667

B15=1.09230558

B16=1.09574779

$\mathrm{B} 17=1.09349685$

$\mathrm{A} 1=65.75176904$

A2 $=32.77074536$

A3 $=122.70171108$

A4 $=122.71041928$

A5 $=111.43966565$ 


$$
\begin{aligned}
& A 6=77.23852207 \\
& A 7=119.12528268 \\
& A 8=105.4088572 \\
& A 9=165.74456334 \\
& A 10=111.11747909 \\
& A 11=110.96853102 \\
& A 12=110.41856521 \\
& A 13=124.32380851 \\
& A 14=111.87762371 \\
& A 15=110.69519899 \\
& A 16=111.45952117 \\
& D 1=-177.83868316 \\
& D 2=-178.70999943 \\
& D 3=177.9184007 \\
& D 4=0.04756144 \\
& D 5=-105.61328698 \\
& D 6=-157.81821772 \\
& D 7=49.19807137 \\
& D 8=141.69798168 \\
& D 9=75.20201757 \\
& D 10=-164.76273295 \\
& D 11=-44.61544335 \\
& D 12=-178.53432222 \\
& D 13=155.63099774 \\
& D 14=-84.9531236 \\
& D 15=34.46987037
\end{aligned}
$$

$\begin{array}{lll}1 & 2 & 3\end{array}$

A

Frequencies -- -333.1074

Red. masses -- $\quad 9.6670$

Frc consts -- 0.6320
A

46.8549

141.4759

1.0323

3.3438

0.0013

0.0394 
IR Inten -- 10.5840

Zero-point correction=

Thermal correction to Energy=

Thermal correction to Enthalpy=

Thermal correction to Gibbs Free Energy=

Sum of electronic and zero-point Energies=

Sum of electronic and thermal Energies=

Sum of electronic and thermal Enthalpies=

Sum of electronic and thermal Free Energies=
0.139879 (Hartree/Particle)

0.149225

0.150169

0.105306
-688.665257
-688.655911
-688.654967
-688.699831

\section{TS 2H-3H-phosphole}

Optimized Parameters !

! (Angstroms and Degrees) !

\begin{tabular}{|c|c|c|c|c|c|}
\hline \multicolumn{2}{|c|}{ ! Name Definition } & \multicolumn{3}{|c|}{ Derivative Info. } & ! \\
\hline ! R1 & $\mathrm{R}(1,4)$ & 1.3906 & $-\mathrm{DE} / \mathrm{DX}=$ & 0.0 & $!$ \\
\hline ! R2 & $\mathrm{R}(1,7)$ & 1.775 & $-\mathrm{DE} / \mathrm{DX}=$ & 0.0 & $!$ \\
\hline ! R3 & $\mathrm{R}(1,15)$ & 1.5073 & $-\mathrm{DE} / \mathrm{DX}=$ & 0.0 & $!$ \\
\hline ! R4 & $\mathrm{R}(2,3)$ & 1.4493 & $-\mathrm{DE} / \mathrm{DX}=$ & 0.0 & $!$ \\
\hline ! R5 & $\mathrm{R}(2,7)$ & 1.7774 & $-\mathrm{DE} / \mathrm{DX}=$ & 0.0 & $!$ \\
\hline ! R6 & $\mathrm{R}(2,8)$ & 1.8867 & $-\mathrm{DE} / \mathrm{DX}=$ & 0.0 & $!$ \\
\hline ! R7 & $\mathrm{R}(2,11)$ & 1.504 & $-\mathrm{DE} / \mathrm{DX}=$ & 0.0 & $!$ \\
\hline ! R8 & $\mathrm{R}(3,4)$ & 1.4162 & $-\mathrm{DE} / \mathrm{DX}=$ & 0.0 & $!$ \\
\hline ! R9 & $\mathrm{R}(3,5)$ & 1.0838 & $-\mathrm{DE} / \mathrm{DX}=$ & 0.0 & $!$ \\
\hline ! R10 & $\mathrm{R}(3,8)$ & 1.8582 & $-\mathrm{DE} / \mathrm{DX}=$ & 0.0 & $!$ \\
\hline ! R11 & $\mathrm{R}(4,6)$ & 1.0851 & $-\mathrm{DE} / \mathrm{DX}=$ & 0.0 & $!$ \\
\hline ! R12 & $\mathrm{R}(8,9)$ & 1.1914 & $-\mathrm{DE} / \mathrm{DX}=$ & 0.0 & $!$ \\
\hline ! R13 & $\mathrm{R}(8,10)$ & 1.0987 & $-\mathrm{DE} / \mathrm{DX}=$ & 0.0 & $!$ \\
\hline ! R14 & $\mathrm{R}(11,12)$ & 1.0918 & $-\mathrm{DE} / \mathrm{DX}=$ & 0.0 & $!$ \\
\hline ! R15 & $\mathrm{R}(11,13)$ & 1.0937 & $-\mathrm{DE} / \mathrm{DX}=$ & 0.0 & $!$ \\
\hline
\end{tabular}




\begin{tabular}{|c|c|c|c|c|c|}
\hline ! R16 & $\mathrm{R}(11,14)$ & 1.0939 & $-\mathrm{DE} / \mathrm{DX}=0$ & 0.0 & $!$ \\
\hline ! R17 & $\mathrm{R}(15,16)$ & 1.0924 & $-\mathrm{DE} / \mathrm{DX}=0$ & 0.0 & $!$ \\
\hline ! R18 & $\mathrm{R}(15,17)$ & 1.0933 & $-\mathrm{DE} / \mathrm{DX}=0$ & 0.0 & $!$ \\
\hline ! R19 & $\mathrm{R}(15,18)$ & 1.0947 & $-\mathrm{DE} / \mathrm{DX}=0$ & 0.0 & $!$ \\
\hline ! A1 & $\mathrm{A}(4,1,7)$ & 112.3116 & $-\mathrm{DE} / \mathrm{DX}=$ & 0.0 & $!$ \\
\hline ! A2 & $\mathrm{A}(4,1,15)$ & 123.6873 & $-\mathrm{DE} / \mathrm{DX}=$ & 0.0 & $!$ \\
\hline ! A3 & $\mathrm{A}(7,1,15)$ & 123.9557 & $-\mathrm{DE} / \mathrm{DX}=$ & 0.0 & $!$ \\
\hline ! A4 & $\mathrm{A}(3,2,7)$ & 110.4158 & $-\mathrm{DE} / \mathrm{DX}=$ & 0.0 & $!$ \\
\hline ! A5 & $\mathrm{A}(3,2,11)$ & 123.0889 & $-\mathrm{DE} / \mathrm{DX}=$ & 0.0 & $!$ \\
\hline ! A6 & $\mathrm{A}(7,2,8)$ & 101.7583 & $-\mathrm{DE} / \mathrm{DX}=$ & 0.0 & $!$ \\
\hline ! A7 & $\mathrm{A}(7,2,11)$ & 125.2554 & $-\mathrm{DE} / \mathrm{DX}=$ & 0.0 & $!$ \\
\hline ! A8 & $\mathrm{A}(8,2,11)$ & 109.0241 & $-\mathrm{DE} / \mathrm{DX}=$ & 0.0 & $!$ \\
\hline ! A9 & $\mathrm{A}(2,3,4)$ & 112.576 & $-\mathrm{DE} / \mathrm{DX}=0$ & 0.0 & $!$ \\
\hline ! A10 & $\mathrm{A}(2,3,5)$ & 122.5187 & $-\mathrm{DE} / \mathrm{DX}=$ & 0.0 & $!$ \\
\hline ! A11 & $\mathrm{A}(4,3,5)$ & 123.9484 & $-\mathrm{DE} / \mathrm{DX}=$ & 0.0 & $!$ \\
\hline ! A12 & $\mathrm{A}(4,3,8)$ & 103.4759 & $-\mathrm{DE} / \mathrm{DX}=$ & 0.0 & $!$ \\
\hline ! A13 & $\mathrm{A}(5,3,8)$ & 105.265 & $-\mathrm{DE} / \mathrm{DX}=$ & 0.0 & $!$ \\
\hline ! A14 & $\mathrm{A}(1,4,3)$ & 113.6881 & $-\mathrm{DE} / \mathrm{DX}=$ & 0.0 & $!$ \\
\hline ! A15 & $\mathrm{A}(1,4,6)$ & 123.62 & $-\mathrm{DE} / \mathrm{DX}=0$ & 0.0 & $!$ \\
\hline ! A16 & $\mathrm{A}(3,4,6)$ & 122.5113 & $-\mathrm{DE} / \mathrm{DX}=$ & 0.0 & $!$ \\
\hline ! A17 & $\mathrm{A}(1,7,2)$ & 90.9568 & $-\mathrm{DE} / \mathrm{DX}=$ & 0.0 & $!$ \\
\hline ! A18 & $\mathrm{A}(2,8,9)$ & 119.2516 & $-\mathrm{DE} / \mathrm{DX}=$ & 0.0 & $!$ \\
\hline ! A19 & $\mathrm{A}(2,8,10)$ & 107.4343 & $-\mathrm{DE} / \mathrm{DX}=$ & 0.0 & $!$ \\
\hline ! A20 & $\mathrm{A}(3,8,9)$ & 122.2931 & $-\mathrm{DE} / \mathrm{DX}=$ & 0.0 & $!$ \\
\hline ! A21 & $\mathrm{A}(3,8,10)$ & 103.7631 & $-\mathrm{DE} / \mathrm{DX}=$ & 0.0 & $!$ \\
\hline ! A22 & $\mathrm{A}(9,8,10)$ & 129.3626 & $-\mathrm{DE} / \mathrm{DX}=$ & 0.0 & $!$ \\
\hline ! A23 & $\mathrm{A}(2,11,12)$ & 111.3834 & $-\mathrm{DE} / \mathrm{DX}=$ & 0.0 & $!$ \\
\hline ! A24 & $\mathrm{A}(2,11,13)$ & 110.8172 & $-\mathrm{DE} / \mathrm{DX}=$ & 0.0 & $!$ \\
\hline ! A25 & $\mathrm{A}(2,11,14)$ & 110.1153 & $-\mathrm{DE} / \mathrm{DX}=$ & 0.0 & $!$ \\
\hline ! A26 & $\mathrm{A}(12,11,13)$ & 108.2106 & $-\mathrm{DE} / \mathrm{DX}=$ & 0.0 & ! \\
\hline A27 & $\mathrm{A}(12,11,14)$ & 108.1166 & $-\mathrm{DE} / \mathrm{DX}=$ & 0.0 & ! \\
\hline
\end{tabular}




\begin{tabular}{|c|c|c|c|c|}
\hline ! A28 & $\mathrm{A}(13,11,14)$ & 108.088 & $-\mathrm{DE} / \mathrm{DX}=0.0$ & $!$ \\
\hline ! A29 & $\mathrm{A}(1,15,16)$ & 111.6274 & $-\mathrm{DE} / \mathrm{DX}=0.0$ & $!$ \\
\hline ! A30 & $\mathrm{A}(1,15,17)$ & 110.9315 & $-\mathrm{DE} / \mathrm{DX}=0.0$ & ! \\
\hline ! A31 & $\mathrm{A}(1,15,18)$ & 111.326 & $-\mathrm{DE} / \mathrm{DX}=0.0$ & 1 \\
\hline ! A32 & $\mathrm{A}(16,15,17)$ & 108.0145 & $-\mathrm{DE} / \mathrm{DX}=0.0$ & ! \\
\hline ! A33 & $\mathrm{A}(16,15,18)$ & 107.2033 & $-\mathrm{DE} / \mathrm{DX}=0.0$ & ! \\
\hline ! A34 & $\mathrm{A}(17,15,18)$ & 107.5445 & $-\mathrm{DE} / \mathrm{DX}=0.0$ & ! \\
\hline ! D1 & $\mathrm{D}(7,1,4,3)$ & 2.0588 & $-\mathrm{DE} / \mathrm{DX}=0.0$ & $!$ \\
\hline ! D2 & $\mathrm{D}(7,1,4,6)$ & 177.2767 & $-\mathrm{DE} / \mathrm{DX}=0.0$ & $!$ \\
\hline ! D3 & $\mathrm{D}(15,1,4,3)$ & -175.5715 & $-\mathrm{DE} / \mathrm{DX}=0.0$ & 1 \\
\hline ! D4 & $\mathrm{D}(15,1,4,6)$ & -0.3536 & $-\mathrm{DE} / \mathrm{DX}=0.0$ & $!$ \\
\hline D5 & $\mathrm{D}(4,1,7,2)$ & -0.7971 & $-\mathrm{DE} / \mathrm{DX}=0.0$ & $!$ \\
\hline ! D6 & $\mathrm{D}(15,1,7,2)$ & 176.8257 & $-\mathrm{DE} / \mathrm{DX}=0.0$ & $!$ \\
\hline ! D7 & $\mathrm{D}(4,1,15,16)$ & -148.579 & $-\mathrm{DE} / \mathrm{DX}=0.0$ & $!$ \\
\hline ! D8 & $\mathrm{D}(4,1,15,17)$ & -28.0593 & $-\mathrm{DE} / \mathrm{DX}=0.0$ & $!$ \\
\hline ! D9 & $\mathrm{D}(4,1,15,18)$ & 91.663 & $-\mathrm{DE} / \mathrm{DX}=0.0$ & $!$ \\
\hline ! D10 & $\mathrm{D}(7,1,15,16)$ & 34.0642 & $-\mathrm{DE} / \mathrm{DX}=0.0$ & $!$ \\
\hline ! D11 & $\mathrm{D}(7,1,15,17)$ & 154.5839 & $-\mathrm{DE} / \mathrm{DX}=0.0$ & 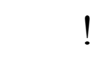 \\
\hline ! D12 & $\mathrm{D}(7,1,15,18)$ & -85.6938 & $-\mathrm{DE} / \mathrm{DX}=0.0$ & $!$ \\
\hline ! D13 & $\mathrm{D}(7,2,3,4)$ & 1.873 & $-\mathrm{DE} / \mathrm{DX}=0.0$ & $!$ \\
\hline ! D14 & $\mathrm{D}(7,2,3,5)$ & 171.0787 & $-\mathrm{DE} / \mathrm{DX}=0.0$ & $!$ \\
\hline ! D15 & $\mathrm{D}(11,2,3,4)$ & -165.9957 & $-\mathrm{DE} / \mathrm{DX}=0.0$ & $!$ \\
\hline ! D16 & $\mathrm{D}(11,2,3,5)$ & 3.21 & $-\mathrm{DE} / \mathrm{DX}=0.0$ & $!$ \\
\hline ! D17 & $\mathrm{D}(3,2,7,1)$ & -0.6125 & $-\mathrm{DE} / \mathrm{DX}=0.0$ & $!$ \\
\hline ! D18 & $\mathrm{D}(8,2,7,1)$ & -69.3624 & $-\mathrm{DE} / \mathrm{DX}=0.0$ & $!$ \\
\hline ! D19 & $\mathrm{D}(11,2,7,1)$ & 166.9357 & $-\mathrm{DE} / \mathrm{DX}=0.0$ & $!$ \\
\hline ! D20 & $\mathrm{D}(7,2,8,9)$ & -144.7306 & $-\mathrm{DE} / \mathrm{DX}=0.0$ & $!$ \\
\hline ! D21 & $\mathrm{D}(7,2,8,10)$ & 14.9556 & $-\mathrm{DE} / \mathrm{DX}=0.0$ & $!$ \\
\hline ! D22 & $\mathrm{D}(11,2,8,9)$ & -10.6693 & $-\mathrm{DE} / \mathrm{DX}=0.0$ & $!$ \\
\hline ! D23 & $\mathrm{D}(11,2,8,10)$ & 149.017 & $-\mathrm{DE} / \mathrm{DX}=0.0$ & $!$ \\
\hline 24 & $\mathrm{D}(3,2,11,12)$ & -169.14 & $-\mathrm{DE} / \mathrm{DX}=0.0$ & $!$ \\
\hline
\end{tabular}




\begin{tabular}{|c|c|c|c|c|}
\hline ! D25 & $\mathrm{D}(3,2,11,13)$ & -48.614 & $-\mathrm{DE} / \mathrm{DX}=$ & 0.0 \\
\hline ! D26 & $\mathrm{D}(3,2,11,14)$ & 70.9233 & $-\mathrm{DE} / \mathrm{DX}=$ & 0.0 \\
\hline ! D27 & $\mathrm{D}(7,2,11,12)$ & 24.8168 & $-\mathrm{DE} / \mathrm{DX}=$ & 0.0 \\
\hline ! D28 & $\mathrm{D}(7,2,11,13)$ & 145.3428 & $-\mathrm{DE} / \mathrm{DX}=$ & $=0.0$ \\
\hline ! D29 & $\mathrm{D}(7,2,11,14)$ & -95.1199 & $-\mathrm{DE} / \mathrm{DX}=$ & 0.0 \\
\hline ! D30 & $\mathrm{D}(8,2,11,12)$ & -95.694 & -DE/DX = & 0.0 \\
\hline ! D31 & $\mathrm{D}(8,2,11,13)$ & 24.832 & $-\mathrm{DE} / \mathrm{DX}=$ & 0.0 \\
\hline ! D32 & $\mathrm{D}(8,2,11,14)$ & 144.3693 & $-\mathrm{DE} / \mathrm{DX}=$ & $=0.0$ \\
\hline ! D33 & $\mathrm{D}(2,3,4,1)$ & -2.5485 & $-\mathrm{DE} / \mathrm{DX}=$ & 0.0 \\
\hline ! D34 & $\mathrm{D}(2,3,4,6)$ & -177.8264 & $-\mathrm{DE} / \mathrm{DX}=$ & 0.0 \\
\hline ! D35 & $\mathrm{D}(5,3,4,1)$ & -171.5741 & $-\mathrm{DE} / \mathrm{DX}=$ & 0.0 \\
\hline ! D36 & $\mathrm{D}(5,3,4,6)$ & 13.148 & $-\mathrm{DE} / \mathrm{DX}=$ & 0.0 \\
\hline ! D37 & $\mathrm{D}(8,3,4,1)$ & 69.2369 & $-\mathrm{DE} / \mathrm{DX}=$ & 0.0 \\
\hline ! D38 & $\mathrm{D}(8,3,4,6)$ & -106.041 & $-\mathrm{DE} / \mathrm{DX}=$ & 0.0 \\
\hline ! D39 & $\mathrm{D}(4,3,8,9)$ & 149.8325 & $-\mathrm{DE} / \mathrm{DX}=$ & 0.0 \\
\hline ! D40 & $\mathrm{D}(4,3,8,10)$ & -8.1787 & $-\mathrm{DE} / \mathrm{DX}=$ & 0.0 \\
\hline ! D41 & $\mathrm{D}(5,3,8,9)$ & 18.4825 & $-\mathrm{DE} / \mathrm{DX}=$ & 0.0 \\
\hline ! D42 & $\mathrm{D}(5,3,8,10)$ & -139.5287 & $-\mathrm{DE} / \mathrm{DX}=$ & 0.0 \\
\hline
\end{tabular}

GradGradGradGradGradGradGradGradGradGradGradGradGradGradGradGradGradGrad

Input orientation:

\begin{tabular}{crrrrc} 
Center & \multicolumn{2}{c}{ Atomic } & \multicolumn{2}{c}{ Atomic } & \multicolumn{2}{c}{ Coordinates (Angstroms) } \\
Number & Number & Type & X & Y & Z \\
- & & & & & \\
1 & 6 & 0 & -0.000122 & 0.000239 & 0.000038 \\
2 & 6 & 0 & -0.000144 & -0.000433 & 2.532862 \\
3 & 6 & 0 & 1.323214 & 0.000226 & 1.941869 \\
4 & 6 & 0 & 1.286563 & 0.032606 & 0.526474
\end{tabular}




\begin{tabular}{|c|c|c|c|c|c|}
\hline 5 & 1 & 0 & 2.222835 & 0.149542 & 2.527619 \\
\hline 6 & 1 & 0 & 2.185148 & 0.003005 & -0.081004 \\
\hline 7 & 15 & 0 & -1.245491 & -0.014235 & 1.264750 \\
\hline 8 & 6 & 0 & 0.753321 & -1.720535 & 2.350540 \\
\hline 9 & 8 & 0 & 1.124303 & -2.299679 & 3.323312 \\
\hline 10 & 1 & 0 & 0.423725 & -2.123415 & 1.383006 \\
\hline 11 & 6 & 0 & -0.248530 & 0.273998 & 3.990624 \\
\hline 12 & 1 & 0 & -1.277867 & 0.040438 & 4.269927 \\
\hline 13 & 1 & 0 & 0.419646 & -0.322894 & 4.617862 \\
\hline 14 & 1 & 0 & -0.067630 & 1.330179 & 4.210771 \\
\hline 15 & 6 & 0 & -0.298043 & -0.071987 & -1.475736 \\
\hline 16 & 1 & 0 & -1.230033 & 0.440935 & -1.723904 \\
\hline 17 & 1 & 0 & 0.505386 & 0.385489 & -2.059229 \\
\hline 18 & 1 & 0 & -0.400046 & -1.109063 & -1.810994 \\
\hline
\end{tabular}

Distance matrix (angstroms):

$\begin{array}{lllll}1 & 2 & 3 & 4 & 5\end{array}$

$1 \mathrm{C} \quad 0.000000$

2 C $2.532824 \quad 0.000000$

$\begin{array}{lllll}3 & \mathrm{C} & 2.349878 & 1.449328 & 0.000000\end{array}$

$\begin{array}{llllll}4 & C & 1.390591 & 2.383757 & 1.416239 & 0.000000\end{array}$

$\begin{array}{lllllll}5 & \mathrm{H} & 3.369347 & 2.228039 & 1.083844 & 2.212433 & 0.000000\end{array}$

$\begin{array}{lllllll}6 & \mathrm{H} & 2.186774 & 3.407023 & 2.198852 & 1.085062 & 2.613008\end{array}$

$\begin{array}{lllllll}7 & \mathrm{P} & 1.775007 & 1.777410 & 2.656491 & 2.637905 & 3.694719\end{array}$

$\begin{array}{lllllll}8 & \mathrm{C} & 3.008920 & 1.886717 & 1.858174 & 2.585550 & 2.384956\end{array}$

$\begin{array}{lllllll}9 & \mathrm{O} & 4.195010 & 2.678754 & 2.690263 & 3.645296 & 2.799747\end{array}$

$\begin{array}{lllllll}10 & \mathrm{H} & 2.569466 & 2.451303 & 2.373028 & 2.475190 & 3.116612\end{array}$

$\begin{array}{lllllll}11 & \mathrm{C} & 4.007671 & 1.504021 & 2.596676 & 3.796724 & 2.874634\end{array}$

$\begin{array}{lllllll}12 & \mathrm{H} & 4.457152 & 2.156767 & 3.491002 & 4.537599 & 3.911836\end{array}$

$\begin{array}{lllllll}13 & \mathrm{H} & 4.648109 & 2.151146 & 2.842847 & 4.197306 & 2.800679\end{array}$

$\begin{array}{llllll}14 \mathrm{H} & 4.416285 & 2.142536 & 2.975086 & 4.134196 & 3.077846\end{array}$ 


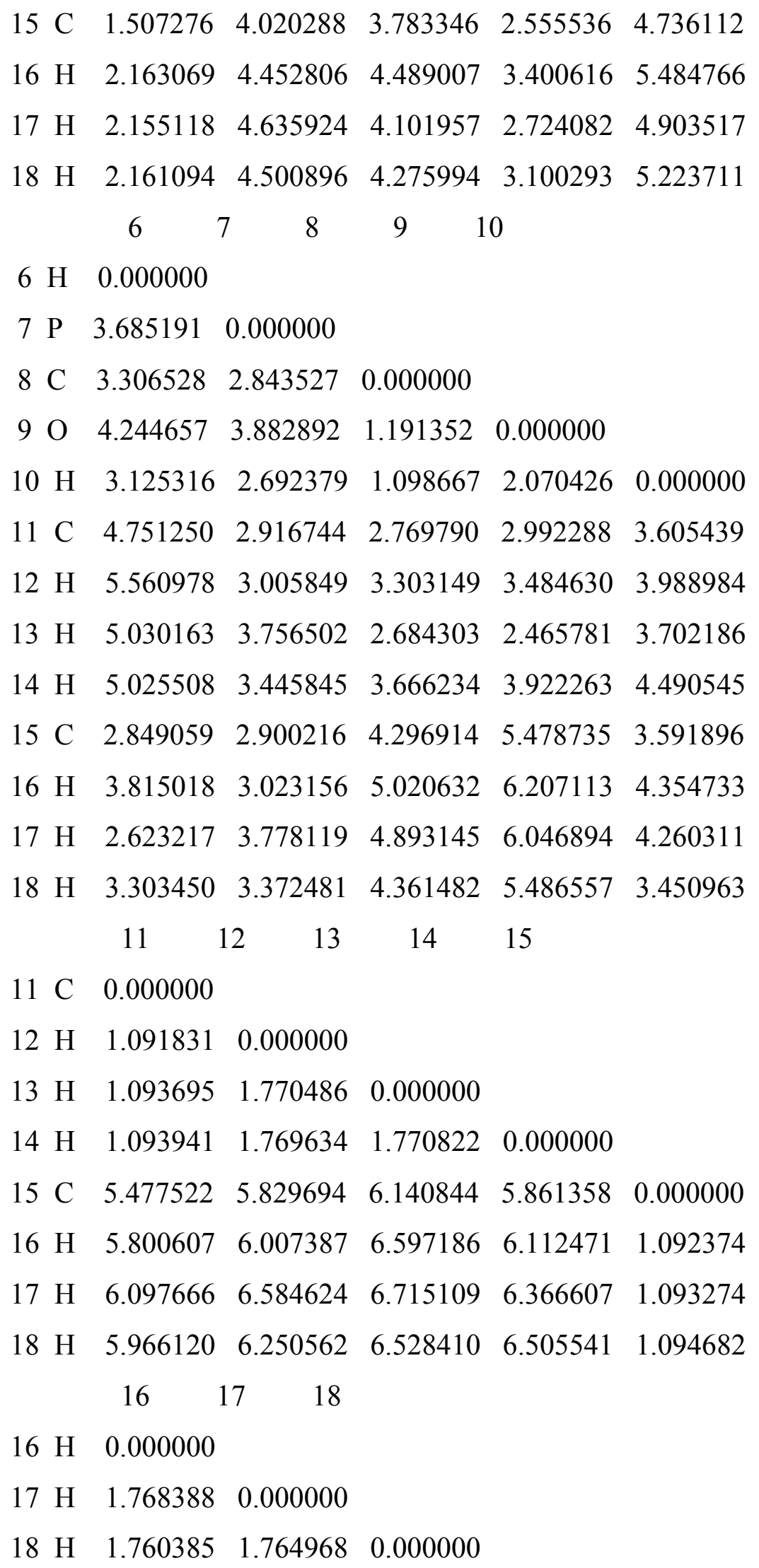


Stoichiometry C7H9OP 12 3

A A

A

Frequencies -- -435.2333 52.7491 132.8753

Red. masses -- 8.5297 1.0282 3.7016

Frc consts -- 0.9520 0.0017 0.0385

IR Inten -- 1.4924 0.1300 2.6935

Zero-point correction $=$ 0.140234 (Hartree/Particle)

Thermal correction to Energy= 0.149515

Thermal correction to Enthalpy= 0.150459

Thermal correction to Gibbs Free Energy=

0.105759

Sum of electronic and zero-point Energies= $-688.658427$

Sum of electronic and thermal Energies= $-688.649146$

Sum of electronic and thermal Enthalpies= $-688.648202$

Sum of electronic and thermal Free Energies= $-688.692902$ 